TI 2017-050/III

Tinbergen Institute Discussion Paper
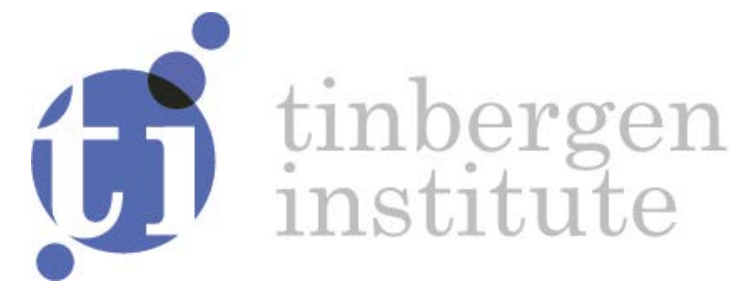

\title{
Smooth Transition Spatial Autoregressive Models
}

Bo Pieter Johannes Andrée ${ }^{1}$

Francisco Blasques ${ }^{1}$

Eric Koomen ${ }^{2}$

${ }^{1}$ VU Amsterdam and Tinbergen Institute

${ }^{2}$ VU Amsterdam 
Tinbergen Institute is the graduate school and research institute in economics of Erasmus University Rotterdam, the University of Amsterdam and VU University Amsterdam.

Contact: discussionpapers@tinbergen.nl

More TI discussion papers can be downloaded at http://www.tinbergen.nl

Tinbergen Institute has two locations:

Tinbergen Institute Amsterdam

Gustav Mahlerplein 117

1082 MS Amsterdam

The Netherlands

Tel.: + $31(0) 205984580$

Tinbergen Institute Rotterdam

Burg. Oudlaan 50

3062 PA Rotterdam

The Netherlands

Tel.: +31(0)10408 8900 


\title{
Smooth Transition Spatial Autoregressive Models
}

\author{
Bo Pieter Johannes Andrée ${ }^{1,2,}$, Francisco Blasques ${ }^{2,3}$, and Eric Koomen ${ }^{1}$ \\ ${ }^{1}$ Department of Spatial Economics/SPINlab, VU Amsterdam, Netherlands \\ ${ }^{2}$ Tinbergen Institute \\ ${ }^{3}$ Department of Econometrics, VU University Amsterdam \\ ${ }^{*}$ Corresponding Author: email b.p.j.andree@vu.nl
}

May 29, 2017

\begin{abstract}
This paper introduces a new model for spatial time series in which cross-sectional dependence varies nonlinearly over space by means of smooth transitions. We refer to our model as the Smooth Transition Spatial Autoregressive (ST-SAR). We establish consistency and asymptotic Gaussianity for the MLE under misspecification and provide additional conditions for geometric ergodicity of the model. Simulation results justify the use of limit theory in empirically relevant settings. The model is applied to study spatio-temporal dynamics in two cases that differ in spatial and temporal extent. We study clustering in urban densities in a large number of neighborhoods in the Netherlands over a 10-year period. We pay particular focus to the advantages of the ST-SAR as an alternative to linear spatial models. In our second study, we apply the ST-SAR to monthly long term interest rates of 15 European sovereigns over 25-year period. We develop a strategy to assess financial stability across the Eurozone based on attraction of individual sovereigns toward the common stochastic trend. Our estimates reveal that stability attained a low during the Greek sovereign debt crisis, and that the Eurozone has remained to struggle in attaining stability since the onset of the financial crisis. The results suggest that the European Monetary System has not fully succeeded in aligning the economies of Ireland, Portugal, Italy, Spain, and Greece with the rest of the Eurozone, while attraction between other sovereigns has continued to increase. In our applications linearity of spatial dependence is overwhelmingly rejected in terms of model fit and forecast accuracy, estimates of control variables improve, and residual correlation is better neutralized.
\end{abstract}

Keywords: Dynamic panel, Threshold models, Spatial heterogeneity, Spatial autocorrelation, Urban density, Interest Rates, Monetary Stability, Sovereign Debt Crisis. 


\section{Introduction}

Spatial entanglement of economic agents plays an important role in the realization of many economic processes measured over space and time. Spatial autocorrelation models are capable of describing the spatial dependence between variables measured across space and are widely adopted in different research fields; see e.g. Kostov (2009) on agricultural land prices, LeSage and Fischer (2008) on regional growth, Baltagi et al. (2014) on housing prices, Hoshino (2016) with an application on crime data, and Debarsy et al. (2015) on foreign direct investments.

Standard spatial models take into account spatial correlation in (un)observed variables, but assume away the possibility of different spatial regimes. In particular, models that allow for spatial (auto)correlation often do not sufficiently relax linearity constraints on functional representations of spatial spillovers. Specifically, spillover-processes are represented by static "global" dependence parameters (Fotheringham, 2009). The literature has stressed the importance of relying on local statistics for spatial dependence instead of global measures due to parameter heterogeneity; see Anselin (1988), Anselin (1995) and Fotheringham (2009).

Typically, spatial aggregation into grouped cross-sectional units relies on the use of econometric tools that are helpful in avoiding ad-hoc sample divisions. Researchers have for example relied on exploratory data analysis Baumont et al. (2006), Geographically Weighted Regression (GWR) (Fotheringham et al., 2002; Su et al., 2012), boosted regression trees (Crase et al., 2012), Bayesian (Glass et al., 2016), nonparametric (Frías and Ruiz-Medina, 2016), or semiparametric (Basile et al., 2014) approaches to model heterogeneity.

All of the above approaches, however, treat the spatial dependence parameter as static. Heterogeneity in interaction is achieved by using spatial trend surfaces or by sample divisions that allow estimation of separate parameter vectors. For example, the Spatial Autoregressive Semiparametric Geoadditive Models discussed by Basile et al. (2014) maintain linearity assumptions with respect to the spatial autoregressive component, but have smooth locally linear dependence structures through a spatial trend surface with respect to exogenous variables.

The GWR approach is a recurring method in spatial econometric literature to model spatial heterogeneity in coefficients. In this approach, neighborhoods are isolated in a Cartesian coordinate system using kernel density functions. Weighted parameter vectors are then estimated for different neighborhoods. Numerous studies pointed out serious drawbacks of this approach. ${ }^{1}$ Additionally, any trend surface strategy requires the entanglement structure

\footnotetext{
${ }^{1}$ Inadequate modeling of spatial lag and error processes (Leung et al., 2000a,b; Fotheringham et al., 2002; Paez et al., 2002a,b), spatial patterns revealed by GWR could be attributed to the procedure itself rather than the data generating process (Wheeler and Tiefelsdorf, 2005; Wheeler, 2007), and finally problems that
} 
to be defined on a two-dimensional Cartesian coordinate system, which could be restrictive for non-geographic weights matrix approaches.

In this paper we propose a parsimonious model that captures heterogeneity in spatial dependence. The model builds on the well-known SAR model (Anselin, 1988) and the Smooth Transition Autoregressive (STAR) framework advocated by Granger and Teräsvirta (1993) and Terasvirta and Anderson (1992); Teräsvirta (1994). The model allows for the possibility of regime-specific dynamics in spillovers and allows for detection of spatial regimes with differential in intensity of spatial spillovers. Feedback loops that amplify spillovers in the cross-section are modeled in this way with varying intensity. The regime-specific dynamics in the cross-sectional dimension are driven by a smooth transition function. The entanglement structure remains exogenously determined through the specification of a spatial weights matrix following standard procedures in the spatial econometric literature. The method provides an attractive alternative to other methods designed to capture crosssectional heterogeneity like the GWR, or models that feature grouped patterns based on fixed-effects. We point out that our method does not depend on spatial trend surfaces and can without difficulty be applied in settings where cross-sectional linkages are based on a broad class of distance metrics.

We establish the asymptotic properties of the intractable Maximum Likelihood Estimator (MLE) for all static parameters. Our asymptotic results allow for possible model misspecification. We focus on $t$-distributed errors as a generalization of Gaussian disturbances and as an attractive way to achieve robustness to outliers or estimate fat-tailed processes. Additional results regarding the ergodicity of data generated by the model are also provided.

We discuss identification of the parameters of the time-varying MLE and the implications for nonlinearity tests based on the Information matrix. As an alternative to Wald-type tests, we explore model selection procedures based on information criteria following Granger et al. (1995); Sin and White (1996). Evidence from the simulation study suggests that we can rule out linear dependence and justify inference on the parameters of the MLE for empirically relevant sample sizes. The simulations also show that the framework is robust to overfitting of additive outliers. False selection rates decrease with sample size but we do not attain a zero false selection rate.

We apply our model empirically in two case studies with different panel dimensions. In the first application, we study dynamics in local residential density in the Netherlands over

relate to bandwidth selection and local violations of least-squares assumptions (Wheeler and Tiefelsdorf, 2005; Farber and Páez, 2007; Cho et al., 2010). We note that grouping observations not by kernels but through fixed effect approaches also has its drawbacks because convergence rates depend on the number of observations in groups tending to infinity (Bonhomme and Manresa, 2015), while in many practical situations additional observations can only be collected over time with group sizes remaining fixed. 
the period 2005 to 2014. The data set consists of a large $N$ and small $T$ panel. We test two hypotheses regarding cross-sectional dependence structures that cannot be captured using linear models: (i) that spatial autoregressive properties should be a magnitude stronger in cities and decay outwards in line with the distance decay of agglomeration effects (Fotheringham, 1981); and (ii) that the relation between urban densities and average household composition in surrounding neighborhoods should vary across the urban gradient, reflecting sorting patterns that arise under single-crossing assumptions about household preferences (Epple and Sieg, 1999). We model nonlinearities in both dependence structures with a threshold function specified around population densities and find strong evidence for both hypotheses.

Our second application is on monthly long term interest rates of 15 European sovereigns over the period 1994-2015. This data set consists of a small $N$ and large $T$ panel. We study the dynamics of endogenously driven contraction toward a common stochastic trend in an integrated network of sovereigns. We pay particular focus on the time-varying properties of local spatial contraction parameters and develop a strategy to assess financial stability. Our spatial weights matrix is based on pair-wise correlations and allows entanglement of areas that are distant from each other from a spatial perspective. This weights matrix specification allows for differential in centrality of the different sovereigns within the spillover network. We explore the dependence structure in the context of an ARMA process, and test several specifications for the nonlinear spatial process based on a mix of the AR and MA components.

In both applications, the results are in overwhelming support of threshold nonlinearities in the spatial dependence parameters. We show that the nonlinear model is able to deliver better in- and out-of-sample forecasts and that the nonlinearities are robust to a wide range of model specifications.

The remainder of this paper is organized as follows. Section 2 considers spatial autocorrelation models and proposes our new framework to capture nonlinear spatial dependencies. Estimation theory for the MLE is examined in Section 3. Section 4 confronts the large sample theory to empirically relevant settings and provides the results of our simulation study. Specifically, we look at the small sample distributions of the MLE and selection frequencies of correctly- and misspecified models. We simulate under innovations drawn from a fat-tailed distribution and explore the impact of additive outliers. The model is applied in Section 5 where we provide results for our analyses on Dutch urban densities and financial stability across the Eurozone. Section 6 summarizes and concludes. Finally, additional results and proofs for our main Theorems are located in Appendix A. 


\section{Linear and Nonlinear Spatial Autoregressive Models}

\subsection{Linear Dynamics: The SAR Model}

Spatial data is often highly dependent across space. In order to model this dependence, Cliff and Ord (1969) proposed the Spatial Autoregressive (SAR) model. The SAR in the context an Autoregressive Moving Average model with Exogenous Regressors (ARMAX) model is given by: ${ }^{2}$

$$
\begin{gathered}
Y_{t}=\rho W Y_{t}+Y_{t-p} \phi+X_{t} \beta+\varepsilon_{t}+\varepsilon_{t-q} \mu \forall t \in \mathbb{Z}, \\
\left\{\varepsilon_{t}\right\}_{t \in \mathbb{Z}} \sim p_{\varepsilon}\left(\varepsilon_{t}, \Sigma, \lambda\right),
\end{gathered}
$$

where $Y_{t}$ denotes a vector of $n$ cross-sectional observations at time $t, \rho$ is the spatial dependence parameter, $W$ is the $n \times n$ matrix of exogenous spatial weights, $\phi$ is a $p \times 1$ vector of autoregressive components, $X_{t}$ is an $n \times(k+1)$ matrix of $k$ exogenous regressors plus a unit vector, $\beta$ is a $(k+1) \times 1$ vector of $k$ coefficients plus an intercept, $\mu$ is a $q \times 1$ vector of MA components and $\varepsilon_{t}$ is the disturbance vector with multivariate density $p_{\varepsilon}\left(\varepsilon_{t}, \Sigma, \lambda\right)$ with zero mean and an unknown $k \times k$ scalar variance-covariance matrix $\Sigma$. Other possible parameters are contained in vector $\lambda$.

In this model structure, each observation of $y_{i t}$ in the vector $Y_{t}$ depends on $k$ individual specific regressors $\left\{x_{i t, k}\right\}_{k=1 t=1}^{K}$, as well as the entries of $\left\{x_{j t, k}\right\}_{k=1 t=1}^{K}{ }^{T}$ for $i \neq j$. For a sufficient amount of observations $n$, such a system cannot be estimated without imposing further restrictions. Spatial dependence modeling is made operational by specifying the spatial weights matrix $W$ as an exogenous function of some relevant normed vector space, for example a function of geographic distances, thereby defining exogenously the structure between cross-sectional entries.

It is standard procedure to row-normalize $W$ such that $\sum_{j=1}^{n} w_{i j}=1 \forall i \in n$, where $w_{i j}$ is the $i, j$-th element from $W$. The parameter $\rho$ captures the spatially weighted effects of neighboring values $W Y_{t}$ on $Y_{t}$. In this simple framework, nonlinear feedback effects across entries can be captured, shown by rewriting the model as:

$$
\begin{gathered}
Y_{t}=H^{-1}\left(Y_{t-p} \phi+X_{t} \beta+\varepsilon_{t}+\varepsilon_{t-q} \mu\right), \\
\left\{\varepsilon_{t}\right\}_{t \in \mathbb{Z}} \sim \mathcal{N} I D\left(0, \sigma_{\varepsilon}^{2}\right),
\end{gathered}
$$

\footnotetext{
${ }^{2}$ Anselin (1988) argues that spatial heterogeneity can further complicated the analysis. His argument is centered on the notion that geographic phenomena often do not deviate around a constant mean, but likely move from one local average to another. For this reason, the simple SAR model may be a problematic model for describing the very phenomenon that Cliff and Ord (1969) are trying to model; see Fotheringham (2009) for a discussion.
} 
where $H:=I_{n}-\rho W$ and $I_{n}$ denotes the $n \times n$ identity matrix. Following LeSage (2008) we obtain the following infinite power series expansion

$$
Y_{t}=\left(I_{n}+\rho^{1: \infty} W^{1: \infty}\right)\left(Y_{t-p} \phi+X_{t} \beta+\varepsilon_{t}+\varepsilon_{t-q} \mu\right),
$$

with $\rho^{1: \infty} W^{1: \infty} V=V+\rho W V+\rho^{2} W^{2} V+\ldots+$, for an arbitrary vector $V$. Equation (3) reveals that the entire ARMAX structure spills over to other regions $j \neq i$ with a rate that declines as proximity to $i$ increases, via the structure imposed by $W$. Feedback effects occur for positive $w_{i j}$ and $w_{j i}$ and mutual neighbours $i$ and $j$, as by construction of the spatial weights matrix $W$, every observation is a second order neighbour of itself. A stable process therefore requires exogenous shocks to die out over space, which for the linear spatial process is guaranteed if $\rho \in\left[1 / \omega_{\min }, 1 / \omega_{\max }\right]$, where $\omega_{\min }$ is the smallest eigenvalue of $W$ (Lee, 2004), or equivalently $|\rho|<1$, if the rows of $W$ sum up to one.

The endogenous nature of this model causes inconsistencies in the least squares estimator that increase with $n$. However, we can consistently estimate the system by Quasi Maximum Likelihood methods (Q)ML or Generalized Methods of Moments (GMM), e.g. Kelejian and Prucha (2010). ML estimation of spatial models with static spatial parameters is pioneered in Ord (1975) and the asymptotics of the QML estimator are derived in Lee (2004). Finite sample distributions are investigated by Das et al. (2003); Bao and Ullah (2007).

\subsection{The Smooth Transition Spatial Autoregressive Model}

The linearity of the SAR model imposes the crucial simplifying assumption that the spatial dependence is fixed for any levels of both $Y_{t}$ and $X_{t}$. As we shall see in Section 5 however, this linearity assumption is not supported by the data. Evidence of nonlinear spatial dependence seems pervasive in economic applications.

In what follows we allow the spatial dependence parameter $\rho$ to change as a function of a vector of variables $Z_{t}$ that may include (spatial lags of) $Y_{t-p}$ or $\varepsilon_{t-q}$ for any $(p, q) \geq 1$ and or $X_{t}$. In particular, we build on the popular smooth transition autoregressive (STAR) model introduced in Terasvirta and Anderson (1992); Teräsvirta (1994). ${ }^{3}$ The resulting Smooth Transition Spatial Autoregressive (ST-SAR) model with ARMAX terms takes the form $^{4}$

$$
Y_{t}=\rho\left(\theta^{\rho} ; Z_{t}\right) \circ W Y_{t}+Y_{t-p} \phi+X_{t} \beta+\varepsilon_{t}+\varepsilon_{t-q} \mu
$$

\footnotetext{
${ }^{3}$ The STAR model is well known in the time-series literature for modeling nonlinear dynamics with thresholds; see Granger and Teräsvirta (1993) for a literature review of nonlinear time-series models. For a comprehensive review of STAR models, the reader is referred to Dijk et al. (2002).

${ }^{4}$ We discuss the nonlinear model within an ARMAX framework because, as we shall see in our applications, all of terms can affect the data both directly and through the spatial dependence parameters. Allowing the terms to explicitly effect $Y_{t}$ directly and through $\rho\left(\theta^{\rho} ; Z_{t}\right)$ simultaneously, is crucial to determine through which channel the effects move.
} 


$$
\left\{\varepsilon_{t}\right\}_{t \in \mathbb{Z}} \sim p_{\varepsilon}\left(\varepsilon_{t}, \Sigma, \lambda\right),
$$

where the spatial dependence $\rho\left(\theta^{\rho} ; Z_{t}\right)$ is determined by

$$
\begin{gathered}
\rho\left(\theta^{\rho} ; Z_{t}\right)=\kappa+\frac{\delta}{1+\exp \left(-\gamma\left(Z_{t}-\tau\left(\theta^{\tau} ; Z_{t}\right)\right)\right)}, \\
\text { and } \tau\left(\theta^{\tau} ; Z_{t}\right)=\alpha+Z_{t} \varphi
\end{gathered}
$$

where $\circ$ denotes element-by-row multiplication, $\theta^{\rho}$ denotes the vector of unknown parameters $\theta^{\rho}:=\left(\kappa, \delta, \gamma, \theta^{\tau}\right)$, and $\theta^{\tau}:=(\alpha, \varphi)$ is a parameter vector of possible additional parameters within the threshold function that may include linear coefficients w.r.t. any of the ARMAX terms contained in $Z_{t}$.

The quantity $Z_{t}-\tau\left(\theta^{\tau} ; Z_{t}\right)$ measures deviations of $Z_{t}$ from a possibly time-varying quantity $\tau\left(\theta^{\tau} ; Z_{t}\right)$. In general, we allow $\tau\left(\theta^{\tau} ; Z_{t}\right)$ to be any function of the data $Z_{t}$. In this paper we consider first order polynomials around three important alternatives, the crosssectional average $\tau\left(\theta^{\tau} ; Z_{t}\right)=\alpha+\varphi n^{-1} \sum_{i=1}^{n} z_{i t}$, the local average $\tau\left(\theta^{\tau} ; Z_{t}\right)=\alpha+\varphi W Z_{t}$, and local observations $\tau\left(\theta^{\tau} ; Z_{t}\right)=\alpha+Z_{t} \varphi$. Other options include modeling $\tau\left(\theta^{\tau} ; Z_{t}\right)$ as a constant $\alpha$ only, or using regional averages $W_{l} Z_{t}$ with $W_{l}$ as a spatial weights matrix that includes up to $l$ higher order spatial lags.

Note that Equation (5)-Equation (6) allow the spatial dependence to change smoothly between regimes. The ST-SAR model differs considerably from time-varying spatial parameter models such as the spatial score model proposed in Blasques et al. (2016) which attempts to filter the unobserved time-varying sequence $\left\{\rho_{t}\right\}_{t \in \mathbb{Z}}$ by means of a score filter. The nonlinear STAR formulation explores the relation between the spatial dependence parameter $\rho$ and variables in $Z_{t}$. In particular, the parameters $\delta, \tau\left(\theta^{\tau} ; Z_{t}\right)$ and $\gamma$ produce dynamics that cannot be reproduced by the time-varying spatial parameter model ofBlasques et al. (2016). It is also worth noting that the STAR dynamics nest not only the linear SAR model of Cliff and Ord (1969), but also, a threshold model (like the TAR model Tong (1978); Tsay (1989); Tong (2015)) with instantaneous switching function between two regimes. The linear SAR case is obtained when $\gamma \rightarrow 0$. In contrast, a threshold switching model is obtained when $\gamma \rightarrow \infty$. Depending on $Z_{t}$, the transition mechanism may be endogenous or exogenous in nature. In the empirical section we shall consider both exogenous cases such as $Z_{t}=X_{t}$ and endogenous examples where we allow the nonlinearities to be driven by ARMA terms.

Finally, we note that, just as in the case of the SAR model, the ST-SAR can also be re-written as

$$
Y_{t}=H\left(\theta^{\rho} ; Z_{t}\right)^{-1}\left(Y_{t-p} \phi+X_{t} \beta+\varepsilon_{t}+\varepsilon_{t-q} \mu\right)
$$

where $H\left(\theta^{\rho} ; Z_{t}\right):=I_{n}-\rho\left(\theta^{\rho} ; Z_{t}\right) \circ W$. 


\section{$3 \quad$ Asymptotic theory for the ST-SAR Model}

Estimation of the ST-SAR model's parameters is crucial to infer if nonlinear dynamics are present in the data. The estimated parameters will also inform us about the existence of threshold dynamics, the location of those thresholds, and the smoothness and speed of transitions. In this section we present and discuss the properties of both the log likelihood function and the ML estimator. We further provide conditions for the existence, strong consistency and asymptotic normality of the MLE for all parameters that define the STSAR. Proofs can be found in Appendix A. Our asymptotic results all refer to increasing the time dimension rather than the spatial dimension since the applications we consider are such that $N$ cannot grow. Additional observations are collected over time only.

Let $\theta$ denote the vector of parameters of our ST-SAR model, $\theta:=\left(\theta^{y}, \theta^{\rho}\right), \theta^{\tau} \in \theta^{\rho}$, $\theta:=(\beta, \phi, \mu, \kappa, \delta, \gamma, \alpha, \varphi)^{\prime}$. Furthermore, let $\theta_{0}$ denote the parameter of interest. Naturally, the ML estimator $\hat{\theta}_{T}$ is defined as

$$
\hat{\theta}_{T} \in \arg \max _{\theta \in \Theta} \sum_{t=1}^{T} \ell_{t}(\theta),
$$

where

$$
\ell_{t}(\theta)=\ln \operatorname{det} H\left(\theta^{\rho} ; Z_{t}\right)+\ln p_{\varepsilon}\left(H\left(\theta^{\rho} ; Z_{t}\right) Y_{t}-Y_{t-p} \phi-X_{t} \beta-\varepsilon_{t-q} \mu, \Sigma ; \lambda\right) .
$$

The dependence of $\ell_{t}(\theta)$ on the data is omitted in the notation for convenience. Equation (9) differs from a standard cross-section likelihood function by the log determinant $\ln \operatorname{det} H\left(\theta^{\rho} ; Z_{t}\right)$, which accounts for the nonlinear spatial feedback (Anselin, 1988). Note that $\varepsilon_{t-q}$ is unobserved and naturally a function of past observed data, with the initial vectors set at zero. In this paper we shall focus on innovations with density $p_{\epsilon}$ given by the multivariate Student's $t$-distribution. The $t$-distribution naturally generalizes the multivariate normal distribution to allow for fat tails, rendering the dynamics more robust to incidental outliers. Using the standard expression for the multivariate $t$-distribution with $\lambda$ degrees of freedom we obtain

$$
\ell_{t}(\theta)=Q\left(\theta^{\rho} ; Z_{t}\right)+A(\theta)+-\frac{1}{2}(\lambda+n) F\left(\theta, Y_{t}, X_{t}, Z_{t}\right),
$$

where $Q\left(\theta^{\rho} ; Z_{t}\right)$ is the $\log$ determinant

$$
Q\left(\theta^{\rho} ; Z_{t}\right):=\ln \operatorname{det} H\left(\theta^{\rho} ; Z_{t}\right)
$$


$A(\theta)$ is a constant given by

$$
A(\theta):=\ln \Gamma((\lambda+n) / 2)\left[\operatorname{det} \Sigma^{\frac{1}{2}}(\lambda \pi)^{\frac{n}{2}} \Gamma(\lambda / 2)\right]^{-1},
$$

and the random element $F\left(\theta, Y_{t}, X_{t}, Z_{t}\right)$ is naturally defined as

$$
F\left(\theta, Y_{t}, X_{t}, Z_{t}\right):=
$$

$\ln \left(1+\lambda^{-1}\left(H\left(\theta^{\rho} ; Z_{t}\right) Y_{t}-\phi Y_{t-1}-X_{t} \beta-\mu \varepsilon_{t-1}\right)^{\prime} \Sigma^{-1}\left(H\left(\theta^{\rho} ; Z_{t}\right) Y_{t}-\phi Y_{t-1}-X_{t} \beta-\mu \varepsilon_{t-1}\right)\right)$.

We first establish the existence and measurability of the MLE $\hat{\theta}_{T}$. This ensures that the $\arg \max$ set in Equation (8) is not empty and that $\hat{\theta}_{T}$ is a random variable.

Assumption 1 (Compactness of $\Theta) \cdot(\Theta, \mathfrak{B}(\Theta))$ is a measurable space and $\Theta$ is a compact subset of $\mathbb{R}^{d_{\theta}}$.

Theorem 1 (Existence and Measurability). Let Assumption 1 hold. Then there exists a.s. an $\mathfrak{\Im} / \mathfrak{B}(\Theta)$-measurable map $\hat{\theta}_{T}: \Omega \rightarrow \Theta$ satisfying Equation (4) for all $T \in \mathbb{N}$.

Consistency of the MLE $\hat{\theta}_{T}$ w.r.t. the parameter of interest $\theta_{0} \in \Theta$ can be obtained under standard regularity conditions. Assumptions 2-3 impose the SE nature of the data and a bounded moment for $Q\left(\theta^{\rho} ; Z_{t}\right)$ and $F\left(\theta, Y_{t}, X_{t}, Z_{t}\right)$. Assumption 4 ensures that $\theta_{0}$ is identified.

Assumption 2. The random sequence $\left\{Y_{t}, X_{t}\right\}_{t \in \mathbb{Z}}$ is $S E$.

Assumption 3. The following moment conditions are satisfied:

$$
\begin{array}{rl}
i & \mathbb{E} \sup _{\theta \in \Theta}\left|Q\left(\theta^{\rho} ; Z_{t}\right)\right|<\infty ; \\
i i & \mathbb{E} \sup _{\theta \in \Theta}\left|F\left(\theta, Y_{t}, X_{t}, Z_{t}\right)\right|<\infty .
\end{array}
$$

Assumption 4. $\theta_{0} \in \Theta$ is the unique maximizer of the limit likelihood; i.e. $\mathbb{E} \ell_{t}\left(\theta_{0}\right)>$ $\mathbb{E} \ell_{t}(\theta) \forall\left(\theta, \theta_{0}\right) \in \Theta \times \Theta: \theta \neq \theta_{0}$.

Theorem 2 below establishes the strong consistency of the MLE $\hat{\theta}_{T}$ with respect to $\theta_{0} \in \Theta$. The moment condition $\mathbb{E} \sup _{\theta \in \Theta}\left|Q\left(\theta^{\rho} ; Z_{t}\right)\right|<\infty$ in Assumption 3 is implied by positive definiteness of $\operatorname{det} H\left(\theta^{\rho} ; Z_{t}\right)^{-1}$ which for the SAR with a row-normalized $W$ follows from $|\rho|<1$. We note that the nonlinear case this is markedly different, and $\left|\rho\left(\theta^{\rho} ; Z_{t}\right)\right|<1$ is not a required condition for $\mathbb{E} \sup _{\theta \in \Theta}\left|Q\left(\theta^{\rho} ; Z_{t}\right)\right|<\infty$; see Lemma 3 in the Supplementary Appendix.

Theorem 2 (Strong consistency). Let Assumptions 1-4 hold. Furthermore, let $\Theta$ be such that $\Sigma$ is positive definite for every $\theta \in \Theta$. Then the MLE satisfies $\hat{\theta}_{T} \stackrel{\text { a.s. }}{\longrightarrow} \theta_{0}$ as $T \rightarrow \infty$. 
Theorem 2 shows that the MLE converges almost surely to $\theta_{0}$ as the sample size increases in the time dimension. When the model is well specified, $\theta_{0}$ corresponds naturally to the so-called true parameter that indexes the distribution of the data. If the model is misspecified, then $\theta_{0}$ is often called a pseudo-true parameter that, by construction, is the minimizer of the Kullback-Leibler divergence (Kullback and Leibler, 1951) between the true conditional probability of the data and the conditional distribution implied by the ST-SAR model; see e.g. White (1994a) for details. In this sense, under model misspecification, the MLE converges at least to the parameter that delivers the best approximation to the true distribution of the data.

Theorem 3 below obtains the asymptotic normality of the MLE by application of Billingsley's central limit theorem (CLT) for an SE martingale difference sequence (mds); see Billingsley (1961). Assumption 5 imposes the mds property. This assumption is appropriate if the model is reasonably well specified. In effect, if the model is well specified, then the score is a mds by construction. This is not the case, however, under strong model misspecification; see White (1994a).$^{5}$

Assumption 5. The score $\left\{\nabla \ell_{t}\left(\theta_{0}\right)\right\}_{t \in \mathbb{Z}}$ is a mds.

Assumption 6 imposes additional moment conditions that ensure the application of a CLT to the score and a uniform law of large numbers to the second derivative of the log likelihood function. Below we let $\nabla^{i} Q\left(\theta_{0}^{\rho} ; Z_{t}\right)$ and $\nabla^{i} F\left(\theta_{0}, Y_{t}, X_{t}, Z_{t}\right)$ denote the $i$ th derivative of $Q\left(\theta_{0}^{\rho} ; Z_{t}\right)$ and $F\left(\theta_{0}, Y_{t}, X_{t}, Z_{t}\right)$ with respect to the vector $\theta$. The moment conditions are imposed on each element of the resulting vectors and matrices.

Assumption 6. The following moment conditions are satisfied:

(i) $\mathbb{E}\left|\nabla Q\left(\theta_{0}^{\rho} ; Z_{t}\right)\right|^{2}<\infty$;

(ii) $\mathbb{E}\left|\nabla F\left(\theta_{0}, Y_{t}, X_{t}, Z_{t}\right)\right|^{2}<\infty$;

(iii) $\mathbb{E} \sup _{\theta \in \Theta}\left|\nabla^{2} Q\left(\theta_{0}^{\rho} ; Z_{t}\right)\right|<\infty$;

(iv) $\mathbb{E} \sup _{\theta \in \Theta}\left|\nabla^{2} F\left(\theta_{0}, Y_{t}, X_{t}, Z_{t}\right)\right|<\infty$.

Theorem 3 delivers the asymptotic Gaussianity of the standardized MLE by imposing the further regularity condition that $\theta_{0}$ lies in the interior of the parameter space $\operatorname{int}(\Theta)$.

Theorem 3 (Asymptotic Normality). Let assumptions 1-6 hold with $\Sigma$ positive definite for every $\theta \in \Theta$. If $\theta_{0} \in \operatorname{int}(\Theta)$, then the $M L E$ satisfies

\footnotetext{
${ }^{5}$ Under strong model misspecification, the asymptotic Gaussianity of the score may still be obtained, under appropriate regularity conditions, by application of a central limit for processes that are near epoch dependent or $L^{p}$ approximable by a mixing process; see e.g. Pötscher and Prucha (1997).
} 


$$
\sqrt{T}\left(\hat{\theta}-\theta_{0}\right) \stackrel{d}{\rightarrow} N\left(0, \mathcal{I}^{-1}\left(\theta_{0}\right) \mathcal{J}\left(\theta_{0}\right) \mathcal{I}^{-1}\left(\theta_{0}\right)\right) \text { as } T \rightarrow \infty,
$$

where $\mathcal{J}\left(\theta_{0}\right):=\mathbb{E} \ell_{t}^{\prime}\left(\theta_{0}\right) \ell_{t}^{\prime}\left(\theta_{0}\right)^{\top}$ is the expectation of the outer product of the score, and $\mathcal{I}^{-1}\left(\theta_{0}\right):=-\mathbb{E} \ell_{t}^{\prime \prime}\left(\theta_{0}\right)$ denotes the Fisher information matrix.

A theory of estimation and inference under correct-specification usually requires a study of the model as a data generating process. We provide additional results regarding the geometric ergodicity of data generated by the ST-SAR in the Supplementary Appendix. As we shall see, the Monte Carlo simulation developed in Section 4 provides evidence of both the consistency and normality claims made in Theorem 2 and Theorem 3 in the correct

and misspecified case. Most importantly, the asymptotic Gaussian distribution seems to provide a good approximation for the finite sample distribution of the MLE in empirically relevant sample sizes.

\section{Monte Carlo study}

To evaluate the adequacy of the ST-SAR framework in fitting dynamic spatial correlation patterns, we conduct a Monte Carlo study. In our first experiment, we investigate whether the MLE is well-behaved and approximately normal for increasing sample sizes. In our second study we investigate model selection based on standard information criteria. To limit the complexity of the experiment, we consider a spatial autoregressive process without exogenous regressors and focus on a model with Student- $t$ innovations. The data generating process is:

$$
Y_{t}=H\left(\theta^{\rho} ; Y_{t-1}\right)^{-1}\left(\varepsilon_{t}\right), \quad \varepsilon_{t} \sim T I D\left(1, I_{n} ; 5\right),
$$

with $\rho\left(\theta^{\rho} ; Y_{t-1}\right)$ as layed out in Equations (5) to (6). In our first experiment we set the parameters values to

$$
\begin{aligned}
& \quad \delta=1.35, \gamma=1.05, \alpha=-0.2, \varphi=1.4, \kappa=-0.4, Z_{t}=Y_{t-1}, \tau\left(\theta^{\tau} ; Z_{t}\right)=\alpha+ \\
& \varphi / N \sum_{1}^{N}\left(Y_{t-1}\right) .
\end{aligned}
$$

The data generating process satisfies the conditions for geometric ergodicity with spatial dependence parameters taking values in $\left|\rho\left(\theta^{\rho} ; Y_{t-1}\right)\right| \in[0,0.95]$ and allows for local positive and negative clustering, see Appendix A.3.

We keep the ratio of distant and close-by neighbors comparable across experiments by allowing the network density of the weights matrix to increase with $N$. In each draw we generate a random zero diagonal row-normalized weights matrix with $N / 10$ neighbors for each observation. The process is initialized with $H_{1}=I_{n}$, and the first 50 steps of the sequence are discarded to avoid dependence on the initialization. We simulate 1000 datasets according to Equation (10) and estimate the parameters of the ST-SAR with $\lambda, \sigma$ and a 
constant as free parameters. We consider samples of size $T=25,100,250$ for $N=30,60$. It is well-known that the small sample behavior of the MLE depends both on the degree of sparseness of the weights matrix and the sample size (Bao and Ullah, 2007). Figure 1 presents kernel density estimates of the distribution of the MLE for the different sample sizes.

Figure 1 presents the results for $N=30$, and shows clearly that for small sample sizes the estimators are not perfectly normal. For larger sample sizes, we see a fast convergence towards the limiting result. The Appendix includes the distributions for $N=60$. The results indicate that for an empirically relevant signal and sample size the MLE is wellbehaved.

Note however that the results do not directly generalize to any empirical setting. Specifically, stronger signals may be estimated appropriately at smaller sample sizes while weaker signals might cause identification problems even in larger samples. We therefore extend our numerical investigation by focussing on selection frequencies based on standard information criteria as a more robust way to conclude on the significance of estimated nonlinearities. We simulate from a weaker dependence signal, and focus on a process driven by local averages to cover the model in a variety of settings. Additionally, the local average should be more severely distorted by additive outliers. We simulate using the following specification:

$1 \delta=0.4, \gamma=1.05, \alpha=-0.2, \varphi=1.4, \kappa=-0.4, Z_{t}=Y_{t-1}, \tau\left(\theta^{\tau} ; Z_{t}\right)=\alpha+\varphi W Y_{t-1}$,

2 SAR with $\rho=0.5$.

We estimate parameters for two versions of the ST-SAR; (i) a parsimonious specification in which the threshold is modeled as constant and the constant spatial dependence component is omitted ( $\varphi$ and $\kappa$ are fixed at 0$)$; and (ii) the correctly specified ST-SAR with all its parameters. We focus on selection between linear and nonlinear models and between the two different nonlinear specifications.

We provide results for the Akaike's information criterion (AIC), the corrected AIC (AICc), and a modified AIC (mAIC). Under well known conditions, the AIC proposed in Akaike $(1973,1974)$ provides a consistent ranking of models based on the Kullback-Leibler divergence between the true distribution of the data and the model-implied distribution. The AICc, introduced by Hurvich and Tsai (1989), improves on the finite sample properties of the AIC; see Brockwell and Davis (1991), McQuarrie (1998) and Burnham and Anderson (2004). The mAIC used here is based on the more general setting put forward by Sin and White (1996).

In this study we also consider the effect of additive outliers, similar to Dijk et al. (1999), 

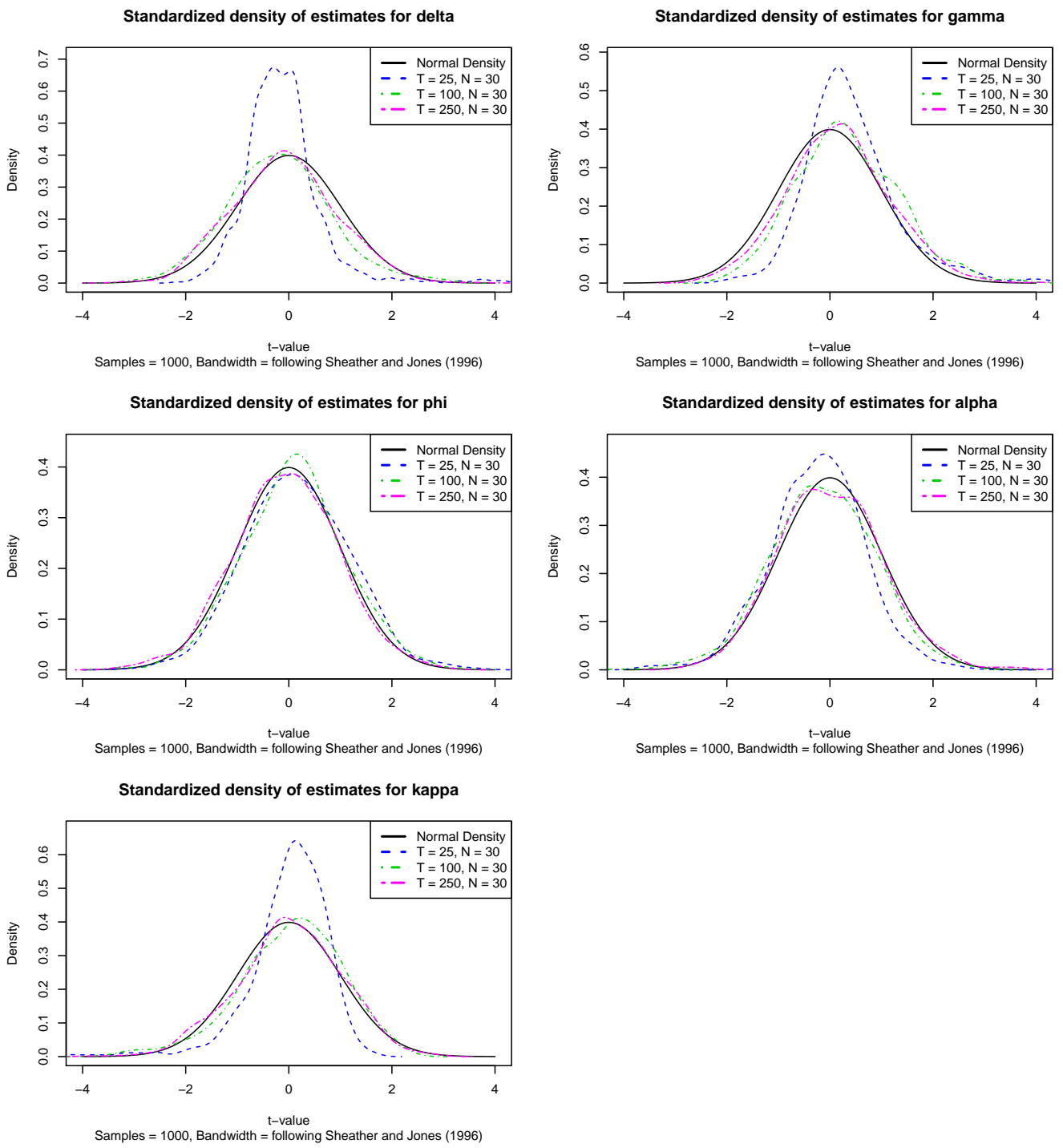

Figure 1: Kernel density estimates of estimated parameters from 1000 simulation replications for $N=30$. 
Table 1: Selection frequencies for data generated from the ST-SAR.

\begin{tabular}{|c|c|c|c|c|c|c|c|c|c|c|}
\hline \multicolumn{2}{|c|}{ ST-SAR DGP } & \multicolumn{3}{|c|}{$\begin{array}{l}\text { ST-SAR } 1 \\
\text { vs. SAR }\end{array}$} & \multicolumn{3}{|c|}{$\begin{array}{l}\text { ST-SAR } 2 \\
\text { vs. SAR }\end{array}$} & \multicolumn{3}{|c|}{$\begin{array}{c}\text { ST-SAR } 2 \\
\text { vs. ST-SAR } 1\end{array}$} \\
\hline & & $\mathrm{AIC}$ & $\mathrm{AICc}$ & mAIC & $\mathrm{AIC}$ & $\mathrm{AICc}$ & $\mathrm{mAIC}$ & AIC & $\mathrm{AICc}$ & $\mathrm{mAIC}$ \\
\hline \multirow[t]{5}{*}{$\mathrm{N}=30$} & $\mathrm{~T}=10$ & 38 & 38 & 38 & 45 & 41 & 43 & 46 & 45 & 46 \\
\hline & $\mathrm{T}=25$ & 62 & 61 & 62 & 63 & 62 & 63 & 50 & 48 & 49 \\
\hline & $\mathrm{T}=50$ & 80 & 79 & 80 & 83 & 82 & 82 & 57 & 57 & 57 \\
\hline & $\mathrm{T}=100$ & 85 & 85 & 85 & 97 & 97 & 97 & 80 & 80 & 80 \\
\hline & $\mathrm{T}=250$ & 100 & 100 & 100 & 100 & 100 & 100 & 96 & 96 & 96 \\
\hline \multirow[t]{5}{*}{$\mathrm{N}=40$} & $\mathrm{~T}=10$ & 51 & 49 & 50 & 52 & 50 & 51 & 44 & 43 & 44 \\
\hline & $\mathrm{T}=25$ & 72 & 72 & 72 & 73 & 72 & 72 & 51 & 50 & 50 \\
\hline & $\mathrm{T}=50$ & 93 & 93 & 93 & 91 & 91 & 91 & 59 & 59 & 59 \\
\hline & $\mathrm{T}=100$ & 92 & 92 & 92 & 100 & 100 & 100 & 84 & 84 & 84 \\
\hline & $\mathrm{T}=250$ & 100 & 100 & 100 & 100 & 100 & 100 & 99 & 99 & 99 \\
\hline \multirow[t]{5}{*}{$\mathrm{N}=50$} & $\mathrm{~T}=10$ & 53 & 52 & 53 & 54 & 52 & 53 & 45 & 43 & 45 \\
\hline & $\mathrm{T}=25$ & 84 & 84 & 84 & 85 & 84 & 85 & 55 & 55 & 55 \\
\hline & $\mathrm{T}=50$ & 98 & 98 & 98 & 98 & 98 & 98 & 66 & 66 & 66 \\
\hline & $\mathrm{T}=100$ & 99 & 99 & 99 & 100 & 100 & 100 & 89 & 89 & 89 \\
\hline & $\mathrm{T}=250$ & 100 & 100 & 100 & 100 & 100 & 100 & 100 & 100 & 100 \\
\hline \multirow[t]{5}{*}{$\mathrm{N}=60$} & $\mathrm{~T}=10$ & 63 & 62 & 63 & 59 & 58 & 59 & 45 & 43 & 44 \\
\hline & $\mathrm{T}=25$ & 88 & 88 & 88 & 87 & 87 & 87 & 57 & 56 & 57 \\
\hline & $\mathrm{T}=50$ & 99 & 99 & 99 & 99 & 99 & 99 & 71 & 71 & 71 \\
\hline & $\mathrm{T}=100$ & 99 & 99 & 99 & 100 & 100 & 100 & 92 & 92 & 92 \\
\hline & $\mathrm{T}=250$ & 100 & 100 & 100 & 100 & 100 & 100 & 100 & 100 & 100 \\
\hline
\end{tabular}

by simulating contaminated sequences according to the following replacement process:

$$
\begin{gathered}
\zeta_{t}=Y_{t}+1 .\left[\mu_{t}>0.5\right] \psi \epsilon_{t}, \\
\left\{\mu_{t}\right\} \sim U I D\left(0, I_{n}\right), \\
\left\{\epsilon_{t}\right\} \sim \operatorname{BID}\left(-I_{n}, I_{n} ; \pi\right),
\end{gathered}
$$

with $\pi=0.05$ and $\psi$ set to the sample equivalents of $\sqrt{\mathbb{E} Y_{t}^{2}-\left(\mathbb{E} Y_{t}\right)^{2}}$, and $\zeta_{t}$ entering the likelihood function.

The results show that both the parsimonious and correctly-specified descriptions of the data are selected over the SAR with increasing frequency as the sample size increases. The parsimonious model seems to fit an important part of the nonlinear process generated under the larger parameter space. When the correctly-specified model is pitted against the parsimonious model, we see that the selection frequency stays moderate for $T \leq 50$, after which it increases rapidly. In large samples the additional information captured by $\hat{\kappa}$ and $\hat{\varphi}$ is decisive in selecting the correct specification with probability 1 .

Table 7 in the Appendix provides results for the contaminated process. Overall, the 
presence of additive outliers has only a small effect. For $T=10$ we observe some increase in power, though for $T>10$ the outliers seem to negatively impact power. Especially the selection frequencies of the correct model seem to go down when pitted against the parsimonious model. While we reach a frequency of $92 \%$ for $(N, T)=(60,100)$ without contamination, we obtain a weaker selection rate of $80 \%$ percent for data distorted by outliers. The reduction in power is somewhat contrary to the univariate framework in which additive outliers are known to sometimes trick the threshold into fitting the contamination incorrectly as a nonlinear process (Dijk et al., 1999). We find that in the cross-sectional case, the results mirror what can be expected in simple linear regression models.

Table 8 provides results for data simulated from the SAR. We narrow our focus to setting the correctly-specified model against the SAR. The results show that the SAR is correctly selected, with increasing probability as sample size grows, over the nonlinear model when the data generating process is in fact linear. Hence, the evidence is in line with the theorized convergence toward zero of false selection probabilities of the nonlinear model, though we do not reach zero even at $N=60$ and $T=250$. At our largest samples, we still maintain around 17 percent false selections. Therefore, one should be careful in interpreting results when the difference in information criteria is small. In our empirical applications however, we find that the difference in information is overwhelming with little room left for chance. Finally, contamination of the linear process does not seem to increase false selection rates.

The study reveals that for empirically relevant sample sizes the MLE is well-behaved and approximately Gaussian. Simulations confirm the appropriateness of standard information criteria that offer different descriptions of spatial spillover processes. The AICc comes forward as the most conservative measure, and therefore we apply it as our primary choice criterion in the empirical section.

\section{The empirics of nonlinear spatial dependencies}

In our empirical study we present two cases. In our first study we use a panel of short $T$ and large $N$. We evaluate nonlinear spatial dynamics in the clustering of Dutch residential densities at the neighborhood level over a period of ten years. The primary focus is on the advantages of the ST-SAR compared to its linear counterpart. We investigate spatially varying features of the dependence structure, particularly in relation to a number of spatially explicit socio-economic variables. The application shows that the ST-SAR is able to fit heterogeneous relationships that the SAR fails to describe. This added flexibility is over-

whelmingly favored by information criteria and forecast accuracy measures, and improves the estimates of the impact of exogenous regressors.

The second application tracks monthly long term interest rates for across an integrated 
network of 15 European sovereigns over a period of 23 years, and explores the ST-SAR in the context of large $T$ and small $N$. The dataset includes observations from before the European Union, during the expansion of the EU, the Great Recession, and the Greek sovereign debt crisis. We develop a strategy to evaluate financial stability by analyzing variation in contraction toward a common trend across the Eurozone. We model contraction in the cross-section with a time-varying threshold function driven by an ARMA specification, and explore convergence and dispersion between rates of different sovereigns over the entire time span. In this application we primarily seek to better understand the temporal dynamics, and show that evidence of nonlinearity is not only pervasive in a sense of statistical significance, but that the estimated dynamics also provide a meaningful description of the economic process.

\subsection{Dutch residential densities}

\subsubsection{Data}

\section{Residential density data}

The Netherlands is one of the most densely populated country in the world. Dutch cities are however relatively small (the largest city has less than one million inhabitants), and population is fairly dispersed in a polycentric network of smaller cores. The sample of observations covers 717 small sized neighborhoods throughout the Randstad, which is the largest urban concentration in The Netherlands. The Randstad includes the four major cities of The Netherlands as well as various mid-sized cities and open spaces. ${ }^{6}$ The Randstad is the major economic area of the Netherlands and the attracting forces of the polycentric network of cities put substantial pressure on remaining open spaces. The heterogeneity in local densities provides an interesting case to apply the ST-SAR model. The time series covers observations from 2005 to 2014 obtained from the Dutch Central Bureaus of Statistics. ${ }^{7}$ An important aspect in the data handling process is harmonization of time-varying boundaries. Throughout the sample period, the Randstad has been further sub-divided in smaller neighborhoods. To facilitate estimation, all observations are standardized to the regional division consistent with 2004 .

The key variable that we focus on is urban density measured as the number of addresses per hectare. We investigate two types on nonlinearities. First, we model nonlinearities in spatial autocorrelation itself to allow for differential strength in clustering. In line with the decay in agglomeration forces along the urban gradient (Fotheringham, 1981), we ex-

\footnotetext{
${ }^{6}$ An overview of the study area with the locations and names of cities is included in the Appendix.

${ }^{7}$ The data is available for download from the Dutch Central Bureau of Statistics: https://www.cbs.nl/nl$\mathrm{nl} /$ dossier/nederland-regionaal/geografische-data
} 
pect autoregressive spatial dependence that captures clustering strength, to fluctuate along with the population densities. The linearity of the SAR implicitly assumes away any variation in autocorrelation along the urban gradient, hence this provides ample opportunity to investigate the advantages of the ST-SAR. The second nonlinearity that we allow is in the relationship between local densities and the spatial average of the share of population under 14 years, which proxies a mixture of social and economic characteristics of the nearby environments. Specifically, dense urban centers accommodate households of a different composition than neighborhoods of lower density. Literature on equilibrium sorting of household types has made strong and empirically tested predictions about the equilibrium distribution of household types across different neighborhoods (Epple and Sieg, 1999). The demand patterns for housing rooted in preference heterogeneity naturally translates into a heterogeneous relationship between density and the household composition in neighborhoods. As households with children locate in low density neighborhoods outside the city center, we can expect that highly dense urban cores have a strong positive relationship with the average share of household with children in surround areas. On the other hand, the below average density of neighborhoods outside the main urban areas produces an inverse relationship. This is specifically interesting to investigate because the linearity of the SAR forces the two opposite relationships to average out which falsely leads to the conclusion that surrounding household composition is a weak correlate of urban density. Hence, this specification allows us to test the ability of the ST-SAR to capture positive and negative relationships simultaneously, and provides an interesting case to contrast its performance with the SAR that can only provide an average description.

Figure 2 shows the strong concentration of urban densities versus the spread out pattern of young population shares that increase outside the high density cores. We regress the local spatial averages of young population against local household densities using a second order queen contiguity weights matrix to capture the average with a relatively wide spatial extent.

\section{Other explanatory variables}

The models include a set of regressors that capture a variety of local demographic and economic characteristics to help explain the observed variation in densities. Furthermore, local and local spatial averages of company densities have been added to capture possible

linkages between working environments and living environments. We followed a Generalto-Specific modeling approach to decide between local observations or spatial averages. All variables are included in the model with a time lag of one period to allow a forecast. 

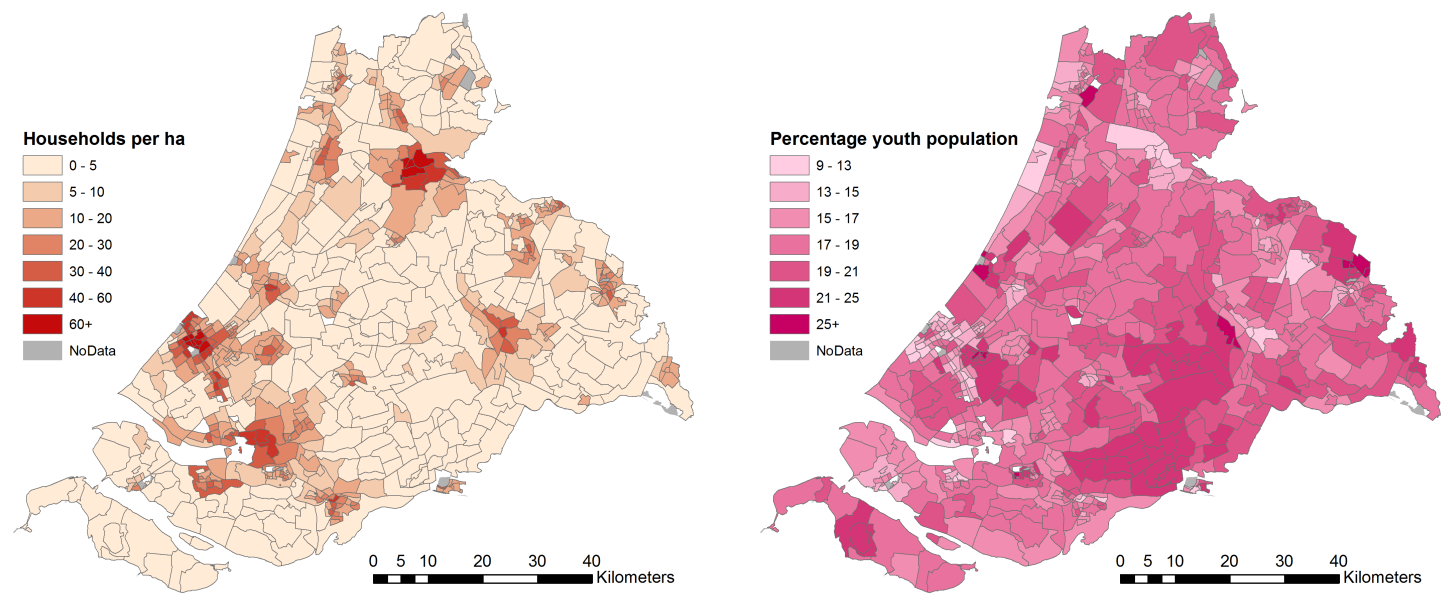

Figure 2: (Left): Distribution of the time average residential density in the Randstad. (Right): distribution of the time average share of population under 14 .

Table 2: Overview of explanatory variables and parameter symbols.

\begin{tabular}{|c|c|c|c|c|}
\hline Parameter & Interacting variable & Units & Range & Mean \\
\hline$\beta_{\text {const }}$ & (Unit vector) & Integer & 1 & 1 \\
\hline$\beta_{c d e n s}$ & Log company density & Continuous & -3.93 to 3.42 & -0.14 \\
\hline$\beta_{w c d e n s}$ & Spatially lagged log company densities & Continuous & -2.62 to 2.30 & -0.21 \\
\hline$\beta_{\% 1 p s h}$ & Percentage of single households & Continuous & 5 to 75.22 & 32.47 \\
\hline$\beta_{w \% 1 p s h}$ & $\begin{array}{l}\text { Spatially lagged percentage single } \\
\text { households }\end{array}$ & Continuous & 0 to 59.75 & 32.15 \\
\hline$\beta_{w \% h h k i d s}$ & $\begin{array}{l}\text { Spatially lagged percentage } \\
\text { households with children }\end{array}$ & Continuous & 0 to 24.11 & 8.75 \\
\hline$\beta_{w \% w i m}$ & $\begin{array}{l}\text { Spatially lagged percentage western } \\
\text { immigrants }\end{array}$ & Continuous & 0 to 59.03 & 36.78 \\
\hline$\beta_{\% \text { nwim }}$ & Percentage non-western immigrants & Continuous & 0 to 67.92 & 9.90 \\
\hline$\beta_{\%>65}$ & Percentage elderly & Continuous & 1 to 43.23 & 15.09 \\
\hline$\beta_{w \%<14}$ & Spatially lagged percentage children & Continuous & 0 to 25.38 & 17.81 \\
\hline$\rho$ & Autoregressive component & Continuous & -.81 to 4.07 & 1.76 \\
\hline$\delta_{i}$ & $\begin{array}{l}\text { Second order queen contiguity spatial } \\
\text { weights matrix }\end{array}$ & $\begin{array}{l}\text { Standardized } \\
\text { Matrix }\end{array}$ & 0 to $46^{*}$ & $18.91^{* *}$ \\
\hline$\gamma_{i}$ & $\begin{array}{l}\text { Log household densities (interacting } \\
\text { with threshold) }\end{array}$ & Continuous & -3.51 to 4.80 & 1.85 \\
\hline$\varphi_{i}$ & Log population densities & Continuous & -2.24 to 5.30 & 2.68 \\
\hline$\kappa_{i}$ & (Unit vector) & Integer & 1 & 1 \\
\hline $\begin{array}{l}\text { Transition } \\
\text { denoted rar } \\
\text { of connectic } \\
\text { connections }\end{array}$ & ons. The eigenvalues range between -.51 to & static spatial & ependence para & $\begin{array}{l}\text { eters. }{ }^{*} \text { Th } \\
\text { hum numbe } \\
\text { hat have } n\end{array}$ \\
\hline
\end{tabular}




\subsubsection{Results for Dutch residential densities}

Table 3 presents the estimation results for the static spatial model, the static spatial autoregressive model with smooth transition nonlinearity in spatially lagged young population shares, and the ST-SAR with additional transition nonlinearity in spatial autocorrelations.

With the static description of spatial linkages, we find strong evidence for spatial dependence of household densities across neighborhoods indicated by the high estimate of $\rho$ and the high t-value. As theorized we find, however, weak evidence for a relationship with the spatial averages of youth population indicated by the small estimate of $\kappa_{w \% 14}$ and its low t-value as the static description averages out over local positive and negative relationships. Household densities are strongly linked to company densities, but the estimates of the control variables have dubious signs. Especially the negative effect of single person households is doubtful, as single households are small and should therefore produce densely populated areas.

The second model allows for smooth transition nonlinearities in the dependence structure with surrounding youth presence. The negative value of $\kappa_{w \% 14-t_{-1}}$ combined with the positive value of $\delta_{w \% 14-t-1}$ signals that the dependence regime of the linkages with surrounding household compositions indeed runs from negative to positive as densities increase. The parameters of the transition function are highly significant while the linear relationship found earlier was fairly weak, revealing that the ST-SAR performs well in fitting the heterogeneity that the SAR fails to describe. The decrease in AICc is substantial (-11939 points), and indicates that the nonlinear specification improves the fitted density drastically. The nonlinear model also improves the estimates for the control variables, both local and surrounding single person households now relate to increasing densities. The effect of company densities is substantially smaller in magnitude, indicating that this impact may be easily overestimated by the SAR. The spatial autocorrelation parameter is still significant but reduced drastically. This suggests that the nonlinearities in the relationship with spatial averages of young population shares may partially capture nonlinearities in the spatial autocorrelations.

Model (3) allows for an additional nonlinear spatial autocorrelation structure, which further decreases the AICc (-1799 points). The mean of the estimated local spatial ST-SAR parameters of model 3 equals 0.116 , which is larger than its static estimate in model (2). The mean of the nonlinear function interacting with the spatial shares of youths equals -0.028 , which is close to the near zero estimate of model (1). 
Table 3: Estimation results for Dutch residential densities from 2005-2014. Significance at 90, 95 and $99 \%$ level are, respectively, indicated as $*, * *$ and $* * *$. $t$-values in parenthesis. Diebold-Mariano tests are one-sided against the previous model, and based on estimates using 2005-2013 data.

\begin{tabular}{|c|c|c|c|}
\hline & $\begin{array}{c}(1) \\
\text { SAR } \\
+ \text { WX } \\
\end{array}$ & $\begin{array}{c}\text { (2) } \\
\text { SAR } \\
+ \text { ST-WX }\end{array}$ & $\begin{array}{c}(3) \\
\text { ST-SAR } \\
+ \text { ST-WX }\end{array}$ \\
\hline$\beta_{\text {const }}$ & $\begin{array}{c}2.309^{* * *} \\
(26.531)\end{array}$ & $\begin{array}{c}0.970^{* * *} \\
(36.611)\end{array}$ & $\begin{array}{c}0.975^{* * *} \\
(41.894)\end{array}$ \\
\hline$\beta_{\text {cdens }_{t-1}}$ & $\begin{array}{l}1.043^{* * *} \\
(201.134)\end{array}$ & $\begin{array}{c}0.108^{* * *} \\
(21.805)\end{array}$ & $\begin{array}{c}0.109^{* * *} \\
(28.381)\end{array}$ \\
\hline$\beta_{w c d e n s_{t-1}}$ & $\begin{array}{c}-0.825^{* * *} \\
(-58.557)\end{array}$ & $\begin{array}{c}-0.101^{* * *} \\
(-13.834)\end{array}$ & $\begin{array}{c}-0.147^{* * *} \\
(-31.390)\end{array}$ \\
\hline$\beta_{\% 1 p s h h_{t-1}}$ & $\begin{array}{c}-0.006^{* * *} \\
(-9.320)\end{array}$ & $\begin{array}{c}0.009^{* * *} \\
(34.365)\end{array}$ & $\begin{array}{c}0.009^{* * *} \\
(38.670)\end{array}$ \\
\hline$\beta_{w \% 1 p s h h_{t-1}}$ & $\begin{array}{c}-0.025^{* * *} \\
(-17.745)\end{array}$ & $\begin{array}{c}0.013^{* * *} \\
(23.710)\end{array}$ & $\begin{array}{c}0.009^{* * *} \\
(19.946)\end{array}$ \\
\hline$\beta_{w \% h h k i d s_{t-1}}$ & $\begin{array}{c}-0.032^{\text {*** }} \\
(-12.355)\end{array}$ & $\begin{array}{c}0.018^{* * *} \\
(17.611)\end{array}$ & $\begin{array}{c}0.015^{* * *} \\
(17.864)\end{array}$ \\
\hline$\beta_{\% \text { nwim }}$-1 & $\begin{array}{c}0.019^{* * *} \\
(35.607)\end{array}$ & $\begin{array}{c}0.003^{* * *} \\
(12.348)\end{array}$ & $\begin{array}{c}0.001^{* * *} \\
(3.170)\end{array}$ \\
\hline$\delta_{w \% 14-t-1}$ & & $\begin{array}{c}1.008^{* * *} \\
(21.324)\end{array}$ & $\begin{array}{c}0.541^{* * *} \\
(25.343)\end{array}$ \\
\hline$\gamma_{w \% 14-t-1}$ & & $\begin{array}{c}0.209^{* * *} \\
(22.501)\end{array}$ & $\begin{array}{c}0.363^{* * * *} \\
(34.370)\end{array}$ \\
\hline$\phi_{w \% 14-t-1}$ & & $\begin{array}{c}1.742^{* * *} \\
(23.416)\end{array}$ & $\begin{array}{c}0.275^{* * *} \\
(9.89)\end{array}$ \\
\hline$\kappa_{w \% 14-t-1}$ & $\begin{array}{c}0.009^{*} \\
(1.987)\end{array}$ & $\begin{array}{c}-0.432^{\text {*** }} \\
(-27.409)\end{array}$ & $\begin{array}{c}-0.387^{* * *} \\
(29.141)\end{array}$ \\
\hline$\delta_{\rho}$ & $\begin{array}{c}0.779 * * * \\
(69.597)\end{array}$ & $\begin{array}{c}0.048^{* * *} \\
(7.113)\end{array}$ & $\begin{array}{c}0.368^{* * *} \\
(35.28) \\
1.235^{* * *}\end{array}$ \\
\hline$\phi_{\rho}$ & & & $\begin{array}{c}(26.499) \\
1.358^{* * *} \\
(84.993)\end{array}$ \\
\hline$\nu$ & $\begin{array}{c}3.013^{* * *} \\
(23.933)\end{array}$ & $\begin{array}{c}2.508^{* * *} \\
(25.772)\end{array}$ & $\begin{array}{c}2.561^{* * *} \\
(26.745)\end{array}$ \\
\hline$\sigma$ & $\begin{array}{c}0.394^{* * *} \\
(30.184)\end{array}$ & $\begin{array}{c}0.192^{* * *} \\
(27.07)\end{array}$ & $\begin{array}{c}0.162^{* * *} \\
(17.358)\end{array}$ \\
\hline$L L$ & -2078.771 & 3893.733 & 4795.447 \\
\hline$A I C c$ & 4179.58 & -7759.407 & -9558.818 \\
\hline$D M_{F E}$ & & $9.920 * * *$ & $1.937^{* *}$ \\
\hline$D M_{S F E}$ & & $4.023^{* * *}$ & 1.093 \\
\hline
\end{tabular}



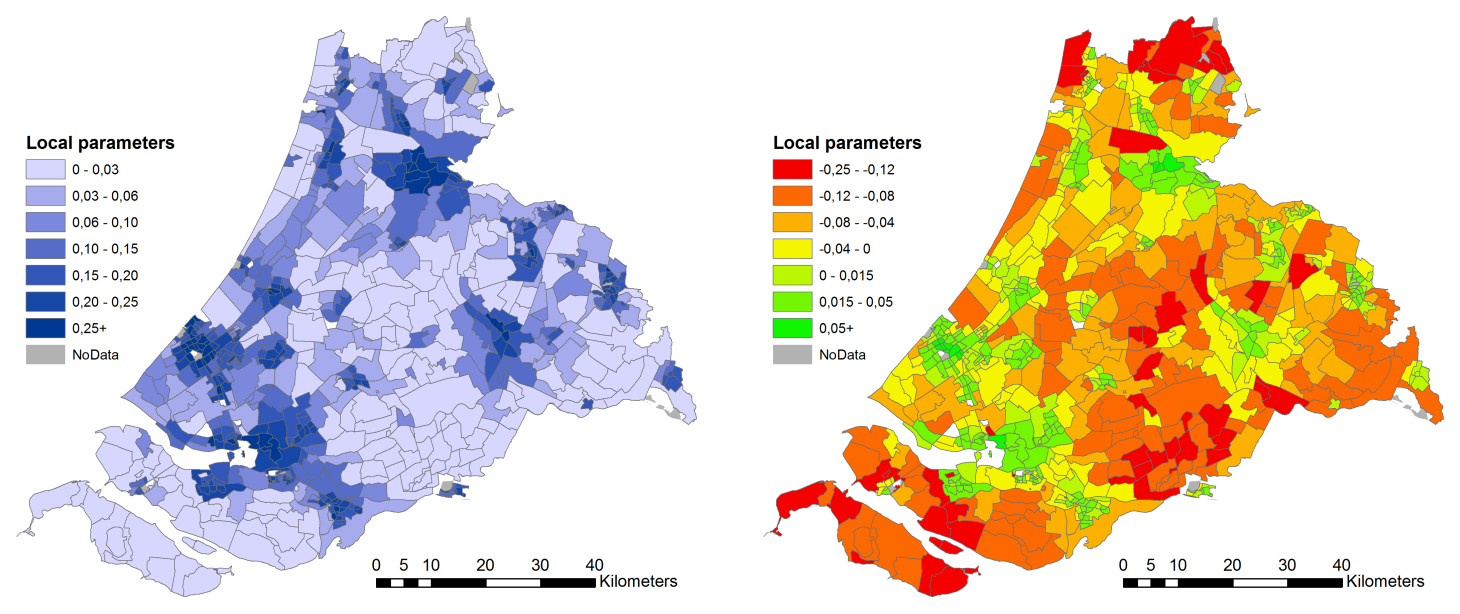

Figure 3: Left): spatial distribution of the time average of estimated autocorrelation parameters. Right): spatial distribution of the time average of estimated dependence on the share on population under 14 years in surrounding neighborhoods.
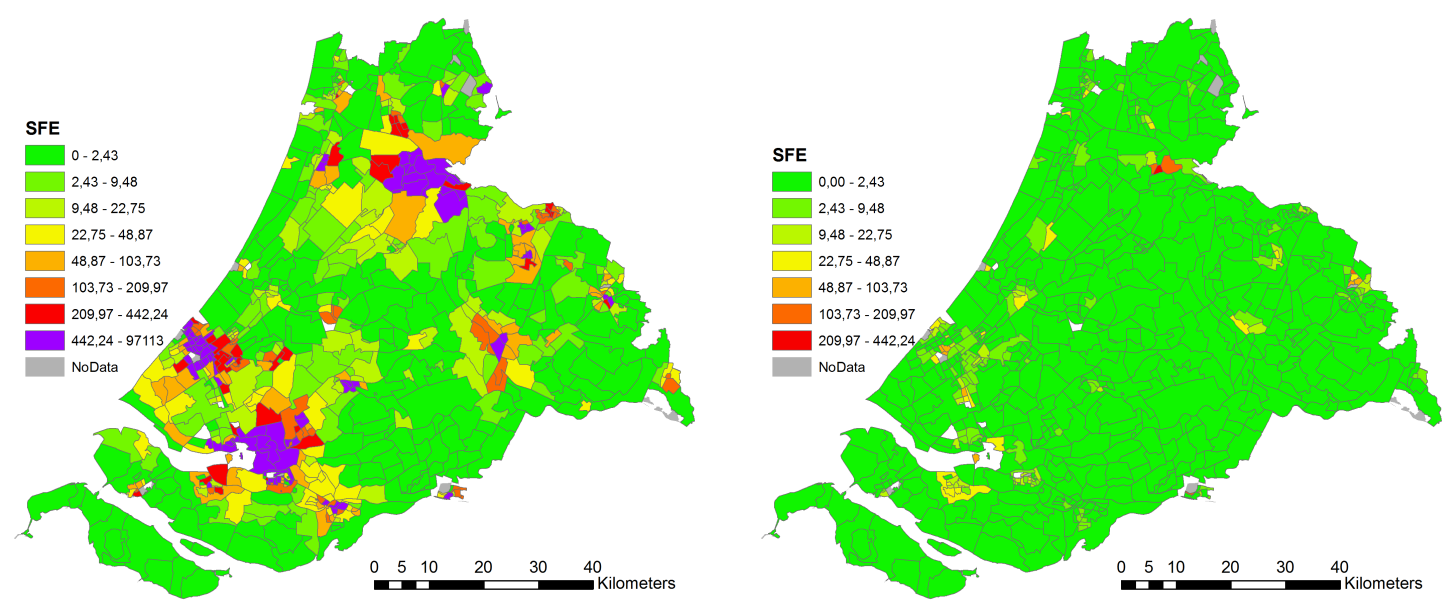

Figure 4: Left): Squared Forecast Errors of the SAR for one-step ahead predictions of the change in the 2014 distribution. Right): Squared Forecast Errors of the ST-SAR based for one-step ahead predictions of the change in the 2014 distribution. Legends are based on natural breaks of the errors of the ST-SAR. 
The maps in fig. 3 show that spatial autocorrelation is high in the urban clusters and decays outwards. The linkages with surrounding youth population shares, move in a similar direction along the urban gradient. Using estimates obtained using the data from 2005-2013, we forecast the distribution of densities for 2014. Diebold-Mariano tests using the SFE for the observations for 2014 are included in table 3 and show that the nonlinear specification not only improves the fitted density but also significantly improves out-of-sample forecasts. The maps in figure 4 compare the changes forecasted by the SAR and the ST-SAR+STWX against the observed changes of 2014. The SFE for the static model clearly reveals a consistent mismatch in major urban areas. The forecast errors of the nonlinear model, however, seem to balance evenly across different areas. This shows that the nonlinear model is better at fitting both rural and urban density processes within one framework. Apart from the clustering of prediction errors, the predictive power across all regions is tremendously improved by the nonlinear model.

\subsection{Financial stability across the Euro region}

In this second empirical study we evaluate the evolution of interest rates on government bonds maturing in ten years for 15 European sovereigns over a period that includes the formation of the European Union, its expansion, the Great Recession and the eventual Greek sovereign debt crisis. Government bonds are essential to the functioning of financial markets and government finances. On the supply side, governments issue debt securities to support spending or finance deficits. On the demand side, institutions and investors use them to manage overall portfolio risk and may require them as part of funding operations. The yields on sovereign debts also play a central role in the pricing and risk-assessment of other financial instruments or investments, because they represent available minimal-risk rates. The yield curves on sovereign debt reflect the overall trust of investors in the stability of economies and provide a policy indicator used by governments to guard the state of their economy.

The Economic and Monetary Union (EMU) comprises a set of policies that aims at converging the economies of the member states of the European Union. The EMU prescribes the euro convergence criteria, comprising the prerequisites for a nation to join the eurozone. Co-movement in the long term interest rates is essential to the monetary stability of the Euro region. Before the European Union, the European Economic Community relied heavily on the European Exchange Rate Mechanism (ERM) to regulate variability in exchange rates of different sovereigns as a way to achieve monetary stability. The ERM played a central role in the preparations for the Economic and Monetary Union and the subsequent introduction of the euro in 1999. The primary goal of the ERM has been to prevent large fluctuations in currency values relative to those of other European sovereigns. Adjustments 
in national interest rates have been at the center of monetary policy used as part of the European Monetary System (EMS) to lower or increase currency value such that the different currencies remained within a narrow range of one another. Replacement of the actual currencies of all participating member states by a common currency mandates that the economies of all member states are relatively in par with one another. After introduction of the euro, national interest rates still play an essential role in ensuring that fluctuation in the economies of all member states remains within a narrow range.

A strong adjustment in long term interest rate of a particular sovereign with respect to the common European average, signals that the underlying economy has difficulty in following the common trend. On the other hand, if all interest rates closely share a common stochastic trend, it signals that economies are in par with on another. The ST-SAR provides a direct method to analyze the strength of convergence toward a common stochastic trend. The average cross-sectional dependence signals the average strength of convergence toward the common, whereas a low average dependence signals that countries are weakly attracted to the common trend. Additionally, low dependence of an individual sovereign signals a potential decoupling of that economy from the common stochastic trend, which may for example result from sustained policy interventions or other local exogenous shocks. The cross-sectional standard deviations in the contraction parameters provides an indicator of the overall financial stability in the Euro region, a high standard deviation indicates that there is substantial difference in the strength of the convergence rates of individual member countries toward the common trend, and a low standard deviation in combination with positive average cross-sectional contraction indicates that the commonality dominates across all individual members. The parameters of the ST-SAR are therefore provide one way to inform us on the functioning of the EMS.

\subsection{European Long term Interest Rates}

We view the interest rates as generated by the model:

$$
V_{t}=c_{t}+Y_{t}
$$

where $V_{t}$ is the observed data vector, $c_{t}$ is the common stochastic trend, and $Y_{t}$ is a vector of dynamics around the common stochastic trend. We are interested it analyzing $Y_{t}$, which contains the contraction and dispersion dynamics around the common stochastics. We assume $c_{t}$ to follow a random walk with $c_{t}=c_{t-1}+v_{t}$, and $\left\{v_{t}\right\}_{t \in \mathbb{Z}} \sim p_{v}\left(v_{t}, \Sigma, \lambda\right)$. Therefore 
our best expectation of $c_{t}$ is $c_{t} \sim \mathbb{E}^{N}\left(Y_{t} \mid Y_{t-1}\right)$, and the dynamics of particular interest are:

$$
Y_{t}=V_{t}-\mathbb{E}^{N}\left(V_{t} \mid V_{t-1}\right)=V_{t}-\mathbb{E}^{N}\left(V_{t-1}\right) \sim V_{t}-N^{-1} \sum_{1}^{N}\left(V_{t-1}\right),
$$

hence we use $Y_{t}=V_{t}-N^{-1} \sum_{1}^{N}\left(V_{t-1}\right)$ as our dependent variable. We refer to $Y_{t}$ as the detrended data. We are interested in a description of the convergence and dispersion dynamics contained in $Y_{t}$ as a nonlinear cross-sectional dependence process driven by the past states of $Y_{t}$ and moving average affects. As a first exploration we regress models of the type: ${ }^{8}$

$$
Y_{t}=H\left(\theta^{\rho} ;\left(Y_{t-p}, \varepsilon_{t-p}\right)\right)^{-1}\left(\varepsilon_{t}\right)
$$

In our extended results, we allow for additional flexibility and explore

$$
Y_{t}=H\left(\theta^{\rho} ;\left(Y_{t-p}, \varepsilon_{t-p}\right)\right)^{-1} g\left(\theta^{g} ; Y_{t-p}, \varepsilon_{t-p}, \varepsilon_{t}\right),
$$

to distinguish between effects that move through the cross-sectional dependence structure or directly though an ARMA structure modeled by $g\left(\theta^{g} ; Y_{t-p}, \varepsilon_{t-p}, \varepsilon_{t}\right)$ with $\varepsilon_{1: p}$ initialized at zero. We explore lags up to order 4, and apply zero restrictions guided by the AICc. As we shall see in our final model, allowing for lags up to order 4 is sufficient to render the residuals free of significant correlations, with some of the parameters restricted to zero. We contrast the models with a linear SAR counterpart. Our spatial structure is based on thresholding of the correlation matrix extracted from the detrended data. This allows for a differential in the centrality of the different sovereigns in the economic network and for entanglement between sovereigns that are distant from each other in a geographic sense but share strong co-movements. We allow each cross-sectional unit to have three neighbors in the network. This number was picked by comparing the AICc of the SAR for networks with respectively 2, 3 and 4 neighbors.

\subsubsection{Long term interest rates data}

We use relative changes (log returns multiplied by 100) of long term interest rates obtained from the OECD, and include observations for 278 months starting October $1993 .{ }^{9}$ The detrended log returns are visualized in fig. 5 , the raw data including a list of labelled events and color codes is provided in Appendix A.3.1. The time series reveal clear common patterns, especially between 1998 and 2008. Before 1998 and after 2008 there are commonalities but

\footnotetext{
${ }^{8}$ The exact threshold specified as $\rho\left(\theta^{\rho} ; Y_{t}, \varepsilon_{t}\right)=\frac{\delta}{1+\exp \left(-\gamma\left(W Y_{t-1}-\left(\alpha+Y_{t-p} \varphi_{\phi}+\varepsilon_{t-p} \varphi_{\mu}\right)\right)\right)}+\kappa$.

${ }^{9}$ https://data.oecd.org/interest/long-term-interest-rates
} 
Table 4: ADF tests without constant on demeaned sequences. Lags are equal across tests to allow a comparison, and determined by estimating $\mathrm{AR}(\mathrm{AICc})$ on the cross-sectional mean. Critical values are not adjusted with a Bonferroni correction.

\begin{tabular}{lcc}
\hline Sovereign & ADF & Stable \\
\hline Austria & -2.227 & stationary \\
Belgium & -2.945 & stationary \\
Germany & -1.382 & nonstationary \\
Denmark & -1.708 & stationary \\
Spain & -1.517 & nonstationary \\
Finland & -1.737 & stationary \\
France & -2.976 & stationary \\
United Kingdom & -1.991 & stationary \\
Greece & -0.805 & nonstationary \\
Ireland & -1.829 & stationary \\
Italy & -1.318 & nonstationary \\
Netherlands & -1.970 & stationary \\
Sweden & -1.887 & stationary \\
Portugal & 0.241 & nonstationary \\
Luxembourg & -1.692 & stationary \\
\hline Cross-sectional mean & -7.815 & stationary \\
\hline
\end{tabular}

specifically the stressed Eurozone sovereigns (Greece, Portugal, Ireland and to some extent Spain and Italy), seem to follow a separate pattern. Table 4 highlights this erratic behavior, and shows that individually, these sovereigns - in addition to Germany that was first to ever hit a negative rate - follow nonstationary trajectories.

\subsubsection{Results using European long term interest rates}

Table 5 contains the estimation results from both the static and nonlinear spatial model for different specifications of the threshold process. The data exhibits very heavy tails, and for simplicity we have fixed $\lambda$ at 2.5 in all models. In the static model, we find strong evidence for spatial dependence in the returns of the rates indicated by the high estimate for $\rho$ together with a small standard error. The three nonlinear specifications, respectively the ST-SAR driven by past observations, moving averages and combinations thereof, all improve the AICc values by several thousand points compared to the SAR, providing ample evidence for nonlinearities in the convergence and dispersion process. The results suggest that the spatial dynamics are driven both by past observations as well as moving averages. On the basis of the reported AICc values, the data clearly favors dynamical spatial dependence. 

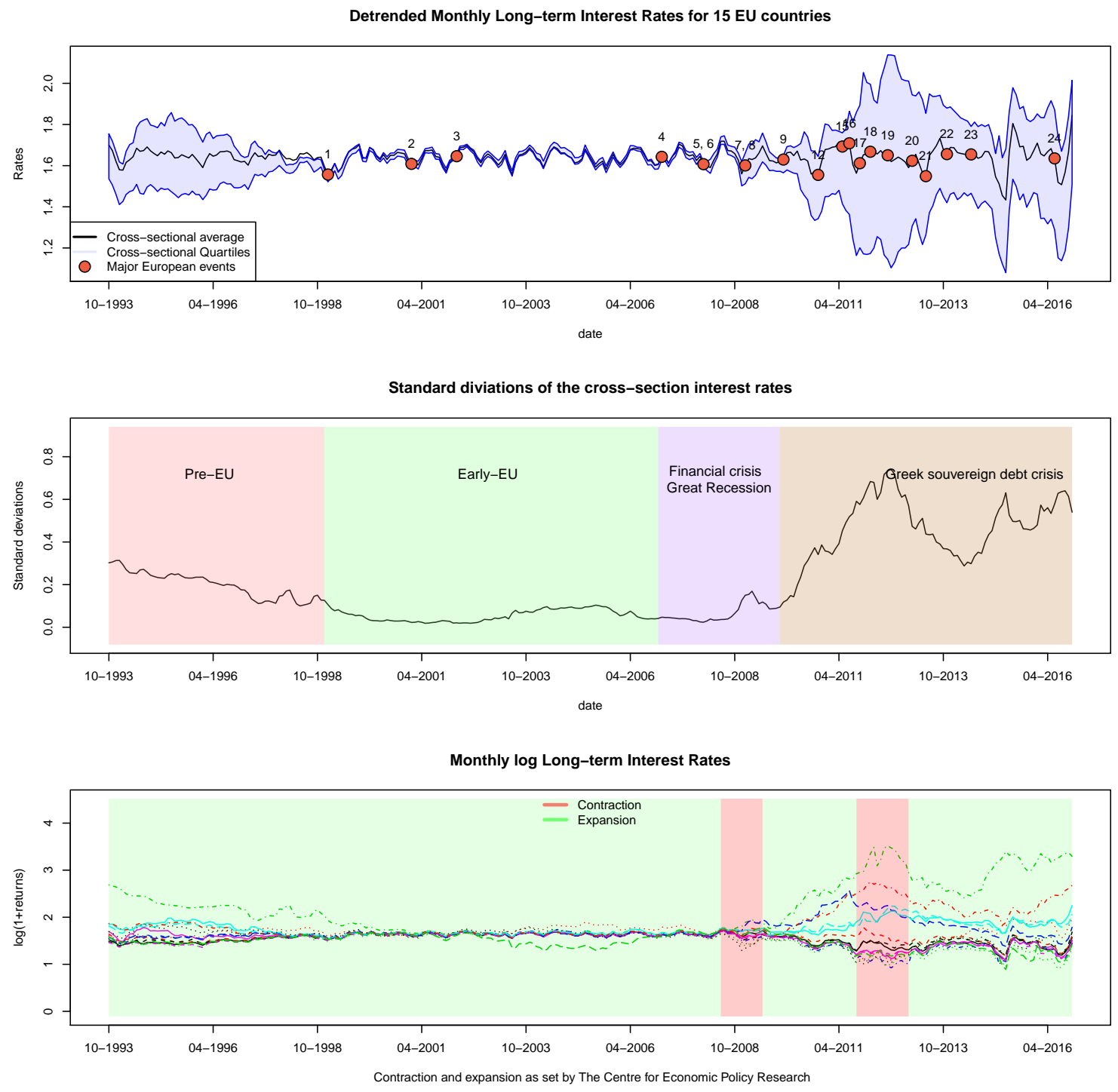

Figure 5: Data on monthly long term interest rates for bonds of 10-year maturity. An overview of te labelled events is contained in Appendix A.3.1. 
However, the SAR contains no time dynamics, which allows the possibility that the nonlinear models attempt to fit time autoregressive forces through the spatial dependence parameters. We therefore extend our analysis by modeling additional dynamics in the time dimension.

\subsubsection{Extensions}

Table 6 presents the estimation results for the models with ARMA effects running through the main time-series process. Again, we observe that the nonlinear spatial model improves the AICc substantially, approximately by -2406 points. The improvement is confirmed by Diebold-Mariano tests on the one-step ahead forecasts, with a $D M=20.01$ for residuals, and $D M=7.37$ for squared residuals. The results provide evidence that the ST-SAR nonlinearities are robust to the specification of different time dynamics, and explain an important part of the variation within the data.

Figure 6 displays the evolution of the fitted spatial dependence parameters. Across the entire time-span, average contraction remained high. A striking feature is the convergence of the spatial parameters in anticipation of the Union, continuing till around 2000. In the pre-EU period we observe two separate regimes. Ireland, Portugal, Italy and Spain form a group that has lower attraction to the common trend, while the remaining sovereigns form a group with a stronger attraction to the common. Greece seems to form an exception and follows an individual trajectory.

After 2000, the parameter sequences corresponding to the different sovereigns nearly linearize. The standard deviations in the parameters remain close to zero for a prolonged period, indicating strong financial stability and near perfect co-movement in the evolution of the individual rates. The onset of the Great Recession around 2008 marks an abrupt turn after which separation in a high and low regime recurs. The standard deviation in attraction parameters attains a peak during the 2012 crisis as rates of stressed sovereigns surge while the remaining nations continue on a downward trend. The months after 2014 are marked by further dispersion. Just before 2015, as Germany hits negative rates for the first time recorded, the range in parameters peaks. Interestingly, the pattern after the recession seems to revert to the pre-EU behavior, with Greece returning to an individual trajectory and Ireland, Portugal, Italy and Spain forming a group of sovereigns that are less bound to the common evolution path. Divergence between the low and high dependence regimes has continued, and the sustained strong variation in contraction parameters indicates that the Eurozone remains to struggle in attaining financial stability since the onset of the financial crisis. The decline in attraction to the common trend for Ireland, Portugal, Italy, and Spain is in sharp contrast to the increase across other member states, and suggests that the EMS has not fully succeeded in aligning these economies of with the rest of the Eurozone, while 
Table 5: Estimation results for the SAR and different specification of the ST-SAR using monthly European LIR data.

\begin{tabular}{|c|c|c|c|c|}
\hline & SAR & $\begin{array}{c}\text { ST-SAR } \\
\quad(\mathrm{AR})\end{array}$ & $\begin{array}{c}\text { ST-SAR } \\
\text { (MA) }\end{array}$ & $\begin{array}{l}\text { ST-SAR } \\
\text { (ARMA) }\end{array}$ \\
\hline$\beta_{\text {const }}$ & \multirow[t]{4}{*}{$\begin{array}{c}0.645^{* * *} \\
(49.47)\end{array}$} & $\begin{array}{c}-0.226^{* * *} \\
(-27.786)\end{array}$ & $\begin{array}{c}0.308^{* * *} \\
(36.12)\end{array}$ & $\begin{array}{c}0.264^{* * *} \\
(31.732)\end{array}$ \\
\hline$\delta_{\rho}$ & & $\begin{array}{l}1.015^{* * *} \\
(101.778)\end{array}$ & $\begin{array}{c}1.081^{* * *} \\
(79.78)\end{array}$ & $\begin{array}{c}1.141^{* * *} \\
(97.392)\end{array}$ \\
\hline$\gamma_{\rho}$ & & $\begin{array}{c}-2.890^{* * *} \\
(-71.282)\end{array}$ & $\begin{array}{c}-0.245^{* * *} \\
(-36.35)\end{array}$ & $\begin{array}{c}-1.696^{* * *} \\
(-51.114)\end{array}$ \\
\hline$\alpha_{\rho}$ & & $\begin{array}{l}.023^{* * *} \\
(2.345)\end{array}$ & $\begin{array}{c}-1.408^{* *} \\
(-12.92)\end{array}$ & $\begin{array}{c}-0.644^{* * *} \\
(-30.204)\end{array}$ \\
\hline$\kappa_{\rho}$ & $\begin{array}{c}0.597^{* * *} \\
(73.84)\end{array}$ & $\begin{array}{l}0.767^{* * *} \\
(115.307)\end{array}$ & $\begin{array}{c}0.457^{* * *} \\
(62.55)\end{array}$ & $\begin{array}{c}0.407 \\
(43.103)\end{array}$ \\
\hline$\varphi_{\phi, t-1}$ & & $\begin{array}{l}0.918^{* * *} \\
(151.192)\end{array}$ & & $\begin{array}{l}1.072^{* * *} \\
(108.303)\end{array}$ \\
\hline$\varphi_{\phi, t-2}$ & & & & $\begin{array}{c}0.021^{*} \\
(2.217)\end{array}$ \\
\hline$\varphi_{\phi, t-3}$ & & $\begin{array}{c}-0.048^{* * *} \\
(-9.267)\end{array}$ & & $\begin{array}{c}0.029 * * * \\
(2.781) \\
0.091^{* * *}\end{array}$ \\
\hline$\varphi_{\phi, t-4}$ & & & & $(9.270)$ \\
\hline$\varphi_{\mu, t-1}$ & & & $\begin{array}{c}14.790^{* * *} \\
(45.66)\end{array}$ & $\begin{array}{c}0.654^{* * *} \\
(28.826)\end{array}$ \\
\hline$\varphi_{\mu, t-2}$ & & & $\begin{array}{c}14.099 * * * \\
(44.85)\end{array}$ & $\begin{array}{c}0.405^{* * *} \\
(16.054)\end{array}$ \\
\hline$\varphi_{\mu, t-3}$ & & & $\begin{array}{c}9.837^{* * *} \\
(40.21)\end{array}$ & $\begin{array}{c}0.404^{* * *} \\
(17.424)\end{array}$ \\
\hline$\varphi_{\mu, t-4}$ & & & $\begin{array}{c}3.802^{* * *} \\
(25.50)\end{array}$ & $\begin{array}{c}0.196^{* * *} \\
(10.235)\end{array}$ \\
\hline$\sigma$ & $\begin{array}{c}0.149 * * * \\
(53.78)\end{array}$ & $\begin{array}{c}0.033^{* * *} \\
(50.362)\end{array}$ & $\begin{array}{c}0.042^{* * *} \\
(53.95)\end{array}$ & $\begin{array}{c}0.029 * * * \\
(53.332)\end{array}$ \\
\hline$L L$ & 2581.47 & 8240.91 & 7562.20 & 9100.78 \\
\hline$A I C c$ & -5156.93 & -16465.78 & -15104.35 & -18173.46 \\
\hline
\end{tabular}


attraction between other sovereigns is currently at an all time high.

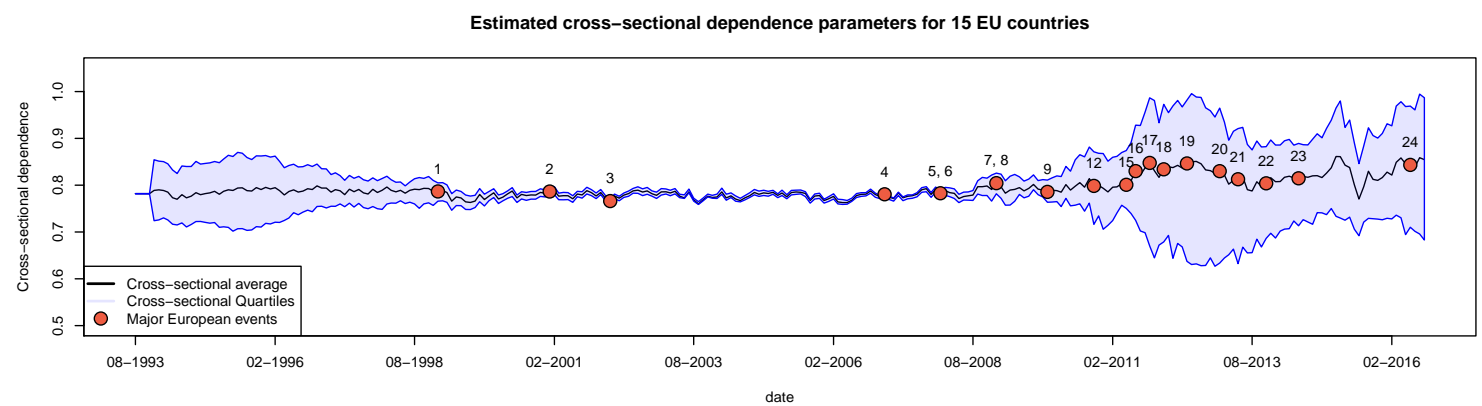

Cross-section standard diviations of the dependence parameters

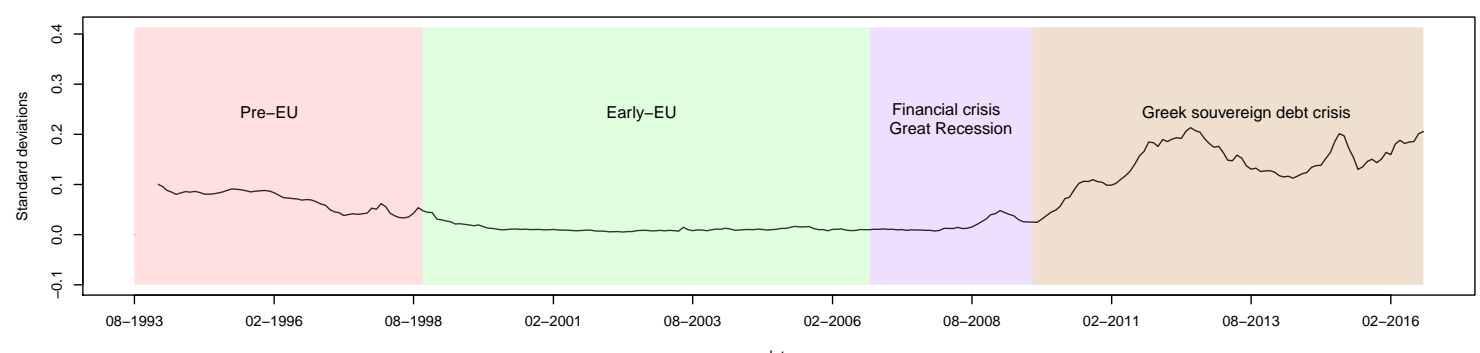

Estimated cross-sectional dependence parameters for $15 \mathrm{EU}$ countries

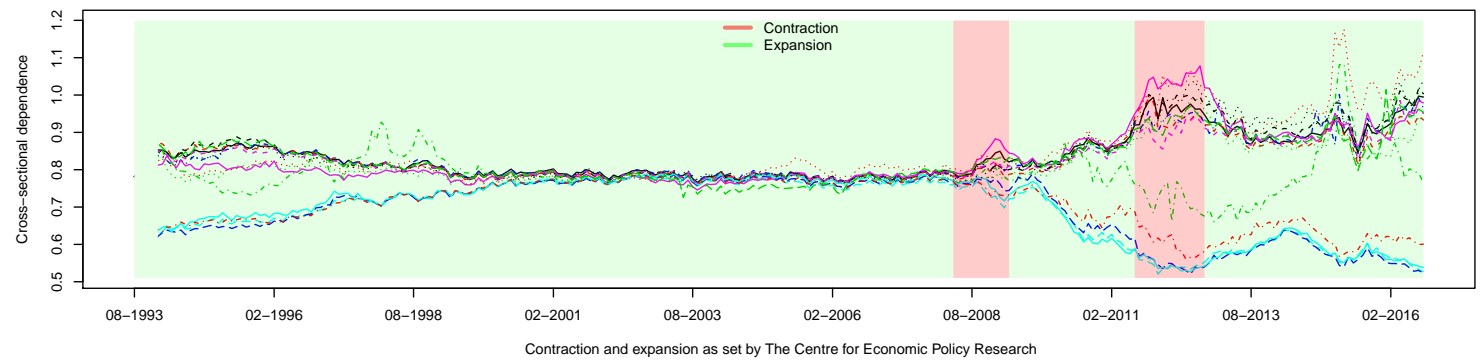

Figure 6: Evolution of spatial parameters estimated with the ST-SAR.

A final noteworthy result is that the residuals of the nonlinear model, as in application our first application, are better centered at zero. Specifically the SAR residuals corresponding to Spain and Greece remain respectively below and above zero for prolonged periods. This can be seen in fig. 10. The residuals of the SAR specification contain significant remaining correlation patterns while the ST-SAR neutralize the dynamics using one autoregressive parameter less in the main equation. This is revealed by the ACF and PACF plotted in fig. 11. Both figures are contained in Appendix A.3.1. 
Table 6: Estimation results for the SAR and different specification of the ST-SAR with additional ARMA parameters using monthly European LIR data.

\begin{tabular}{|c|c|c|}
\hline & $\begin{array}{l}\mathrm{SAR}+ \\
\mathrm{ARMA}\end{array}$ & $\begin{array}{c}\text { ST-SAR (ARMA) } \\
+ \text { ARMA }\end{array}$ \\
\hline$\beta_{\text {const }}$ & $\begin{array}{c}0.004 \\
(0.176)\end{array}$ & $\begin{array}{l}-.848^{* * *} \\
(-55.761)\end{array}$ \\
\hline$\phi_{t-1}$ & $\begin{array}{c}-0.185^{* * *} \\
(-16.124)\end{array}$ & $\begin{array}{c}0.154^{* * *} \\
(3.255)\end{array}$ \\
\hline$\phi_{t-2}$ & $\begin{array}{c}0.048^{* * *} \\
(6.557)\end{array}$ & \\
\hline$\phi_{t-3}$ & $\begin{array}{c}0.230^{* * *} \\
(18.707)\end{array}$ & $\begin{array}{c}0.281^{* * *} \\
(4.062)\end{array}$ \\
\hline$\phi_{t-4}$ & $\begin{array}{l}0.238^{* * *} \\
(21.479)\end{array}$ & $\begin{array}{c}0.301^{* * *} \\
(5.079)\end{array}$ \\
\hline$\mu_{t-1}$ & $\begin{array}{l}1.632^{* * *} \\
(76.744)\end{array}$ & $\begin{array}{l}.932^{* * *} \\
(10.213)\end{array}$ \\
\hline$\mu_{t-2}$ & $\begin{array}{l}1.554^{* * *} \\
(55.753)\end{array}$ & $\begin{array}{c}0.454^{* * *} \\
(3.555)\end{array}$ \\
\hline$\mu_{t-3}$ & $\begin{array}{l}0.938^{* * *} \\
(38.140)\end{array}$ & $\begin{array}{c}0.463^{* * *} \\
(6.463)\end{array}$ \\
\hline$\mu_{t-4}$ & $\begin{array}{c}0.234^{* * *} \\
(16.832)\end{array}$ & $\begin{array}{l}0.042^{* *} \\
(3.112)\end{array}$ \\
\hline$\delta_{\rho}$ & & $\begin{array}{c}6.427 * * \\
(2.440)\end{array}$ \\
\hline$\gamma_{\rho}$ & & $\begin{array}{c}-1.468^{* * *} \\
(-24.109)\end{array}$ \\
\hline$\alpha_{\rho}$ & & $\begin{array}{c}-0.813^{* *} \\
(-2.258)\end{array}$ \\
\hline$\kappa_{\rho}$ & $\begin{array}{c}0.666^{* * *} \\
(64.837)\end{array}$ & $\begin{array}{c}0.438^{* * *} \\
(51.156)\end{array}$ \\
\hline$\varphi_{\phi, t-1}$ & & $\begin{array}{l}0.915^{* * *} \\
(16.250)\end{array}$ \\
\hline$\varphi_{\phi, t-2}$ & & \\
\hline$\varphi_{\phi, t-3}$ & & $\begin{array}{c}-0.290 * * * \\
(-3.398)\end{array}$ \\
\hline$\varphi_{\phi, t-4}$ & & $\begin{array}{c}-0.323^{* * *} \\
(-4.359)\end{array}$ \\
\hline$\varphi_{\mu, t-1}$ & & $\begin{array}{c}-.804^{* * *} \\
(-7.003)\end{array}$ \\
\hline$\varphi_{\mu, t-2}$ & & $\begin{array}{c}-0.385^{* *} \\
(-2.514)\end{array}$ \\
\hline$\varphi_{\mu, t-3}$ & & $\begin{array}{c}-0.490 * * * \\
(-6.196)\end{array}$ \\
\hline$\varphi_{\mu, t-4}$ & & \\
\hline$\sigma$ & $\begin{array}{c}0.045^{* * *} \\
(54.165)\end{array}$ & $\begin{array}{l}0.028^{* * *} \\
(55.275)\end{array}$ \\
\hline$L L$ & 7932.76630 & 9517.539 \\
\hline$A I C c$ & -16590.74 & -18996.89 \\
\hline
\end{tabular}




\section{Conclusion}

In this paper we introduced a new model for nonlinear spatial time series in which crosssectional dependence varies smoothly over space by means of a specification that allows for smooth-transitions between multiple dependence regimes. In this framework, nonlinearities in cross-sectional dynamics are directly modeled as a function of the data. This is an advance over existing methods that rely on ad-hoc sample divisions, ambiguous dummy specifications, or weighted parameter vectors derived from geographic kernel density estimates. All whom are known to have serious drawbacks. Allowing for time-variation is particularly useful when modeling spatial contraction over long time periods - it cannot be assumed that spatial dependence remains fixed for prolonged periods. In addition, the nonlinearities over the cross-section are particularly useful if $N$ is large - as in our first application - or when dispersion and convergence dynamics are key features of the data as in our second application.

We have shown that the parameters of the model can be consistently estimated by maximum likelihood under appropriate regularity conditions. In particular, we provide conditions that deliver existence, strong consistency and asymptotic normality of the MLE of all static parameters that constitute the dynamic dependence structure. The theory holds for both correctly specified and misspecified models. Additional results made available in a Supplementary Appendix deliver the geometric ergodicity of data generated by the ST-SAR model. Our simulation evidence suggests that the limit theory is relevant and that the ML estimator behaves well in finite samples. Furthermore, we find that standard information criteria are able to distinguish between the SAR specification and ST-SAR type nonlinearities. The simulations results showed that model selection is robust to overfitting of additive outliers.

Our empirical applications on Dutch household densities and European long term interest rates, provide ample evidence that spatial nonlinearities are not only persistent, but also explain a major part of the observed dynamics. Across our applications we find that ST-SAR improves in- and out-of-sample forecasts, reduces AICc, and better neutralizes residual correlation. We find that spatial dependence in is driven both exogenously and endogenously, and reacts to the slow assimilation of exogenous shocks. 


\section{References}

\section{References}

Akaike, H. (1973). Information theory and an extension of the maximum likelihood principle. Technical report.

Akaike, H. (1974). A new look at the statistical model identification. IEEE Transactions on Automatic Control, 19(6):716-723.

Anselin, L. (1988). Spatial Econometrics: Methods and Models.

Anselin, L. (1995). Local Indicators of Spatial Association-LISA. Geographical Analysis, $27(2): 93-115$.

Baltagi, B. H., Fingleton, B., and Pirotte, A. (2014). Spatial lag models with nested random effects: An instrumental variable procedure with an application to English house prices. Journal of Urban Economics, 80:76-86.

Bao, Y. and Ullah, A. (2007). Finite sample properties of maximum likelihood estimator in spatial models. Journal of Econometrics, 137(2):396-413.

Basile, R., Durbán, M., Mínguez, R., María Montero, J., and Mur, J. (2014). Modeling regional economic dynamics: Spatial dependence, spatial heterogeneity and nonlinearities. Journal of Economic Dynamics and Control, 48:229-245.

Baumont, C., Ertur, C., and Gallo, J. L. (2006). The European Regional Convergence Process, 1980-1995: Do Spatial Regimes and Spatial Dependence Matter? International Regional Science Review, 29(1):1-41.

Billingsley, P. (1961). The Lindeberg-Levy Theorem for Martingales. Proceedings of the American Mathematical Society, 12(5):788.

Blasques, F., Koopman, S. J., Lucas, A., and Schaumburg, J. (2016). Spillover dynamics for systemic risk measurement using spatial financial time series models. Journal of Econometrics, 195(2):211-223.

Bonhomme, S. and Manresa, E. (2015). Grouped Patterns of Heterogeneity in Panel Data. Econometrica, 83(3):1147-1184.

Brockwell, P. J. and Davis, R. A. (1991). Time Series: Theory and Methods. Springer.

Burnham, K. P. and Anderson, D. R. (2004). Multimodel inference: understanding aic and bic in model selection. Sociological Methods 83 Research, 33:261-304. 
Cho, S.-H., Lambert, D. M., and Chen, Z. (2010). Geographically weighted regression bandwidth selection and spatial autocorrelation: an empirical example using Chinese agriculture data. Applied Economics Letters, 17(8):767-772.

Cliff, A. and Ord, J. (1969). The Problem of Spatial Autocorrelation. In A. J. Scott, editor, In London Papers in Regional Science, pages 25-55. Pion, London.

Cline, D. B. H. and Pu, H.-M. H. (1998). Verifying irreducibility and continuity of a nonlinear time series. Statistics \& Probability Letters, 40:139-148.

Cline, D. B. H. and Pu, H.-M. H. (1999). Geometric Ergodicity of Nonlinear Time Series. Statistica Sinica, 9:1103-1118.

Comte, F. and Lieberman, O. (2003). Asymptotic theory for multivariate GARCH processes. Journal of Multivariate Analysis, 84(1):61-84.

Crase, B., Liedloff, A. C., and Wintle, B. A. (2012). A new method for dealing with residual spatial autocorrelation in species distribution models. Ecography, 35(10):879-888.

Das, D., Kelejian, H. H., and Prucha, I. R. (2003). Finite sample properties of estimators of spatial autoregressive models with autoregressive disturbances. Papers in Regional Science, 82(1):1-26.

Debarsy, N., Jin, F., and Lee, L.-f. (2015). Large sample properties of the matrix exponential spatial specification with an application to FDI. Journal of Econometrics, 188(1):1-21.

Dijk, D., Teräsvirta, T., and Franses, P. (2002). Smooth transition autoregressive models - a survey of recent developments. Econometric Reviews, (August 2013):37-41.

Dijk, D. V., Franses, P. H., Lucas, A., and Lucas, A. (1999). Testing for Smooth Transition Nonlinearity in the Presence of Outliers. Journal of Business 85 Economic Statistics, $17(2): 217$.

Dudley, R. M. (2002). Real Analysis and Probability. Cambridge Studies in Advanced Mathematics. Cambridge University Press.

Epple, D. and Sieg, H. (1999). Estimating Equilibrium Models of Local Jurisdictions. The Journal of Political Economy, 10(2):130-153.

Farber, S. and Páez, A. (2007). A systematic investigation of cross-validation in GWR model estimation: Empirical analysis and Monte Carlo simulations. Journal of Geographical Systems, 9(4):371-396. 
Foland, G. B. (2009). A Guide to Advanced Real Analysis. Dolciani Mathematical Expositions. Cambridge University Press.

Fotheringham, A. S. (1981). Spatial structure and distance-decay parameters. Annals of the Association of American Geographers, 71(3):425-436.

Fotheringham, A. S. (2009). "The Problem of Spatial Autocorrelation" and Local Spatial Statistics. Geographical Analysis, 41(4):398-403.

Fotheringham, A. S., Brunsdon, C., and Charlton, M. (2002). Geographically Weighted Regression: The Analysis of Spatially Varying Relationships.

Frías, M. and Ruiz-Medina, M. (2016). Wavelet nonparametric estimation from strong spatial correlated high-dimensional data. Spatial Statistics, 18:363-385.

Gallant, R. and White, H. (1988). A Unified Theory of Estimation and Inference for Nonlinear Dynamic Models. Cambridge University Press.

Glass, A. J., Kenjegalieva, K., and Sickles, R. C. (2016). A spatial autoregressive stochastic frontier model for panel data with asymmetric efficiency spillovers. Journal of Econometrics, 190(2):289-300.

Granger, C. W., King, M. L., and White, H. (1995). Comments on testing economic theories and the use of model selection criteria. Journal of Econometrics, 67(1):173-187.

Granger, W. J. and Teräsvirta, T. (1993). Modelling Nonlinear Economic Relationships. Oxford University Press, 61(4):1241-1243.

Hoshino, T. (2016). Semiparametric Spatial Autoregressive Models with Endogenous Regressors: With an Application to Crime Data. Journal of Business 85 Economic Statistics, pages $1-51$.

Hurvich, C. and Tsai, C.-L. (1989). Regression and time series model selection in small samples. Biometrika, 76:297-307.

Kelejian, H. H. and Prucha, I. R. (2010). Specification and estimation of spatial autoregressive models with autoregressive and heteroskedastic disturbances. Journal of Econometrics, 157(1):53-67.

Kostov, P. (2009). A Spatial Quantile Regression Hedonic Model of Agricultural Land Prices. Spatial Economic Analysis, 4(1):53-72.

Krengel, U. (1985). Ergodic theorems. Walter de Gruyter. 
Kullback, S. and Leibler, R. A. (1951). On Information and Sufficiency. The Annals of Mathematical Statistics, 22(1):79-86.

Lange, K. (1999). Numerical analysis for statisticians. Springer.

Lee, L. F. (2004). Asymptotic distributions of quasi-maximum likelihood estimators for spatial autoregressive models.

LeSage, J. P. (2008). An Introduction to Spatial Econometrics. Revue d'économie industrielle, 123(123):19-44.

LeSage, J. P. and Fischer, M. M. (2008). Spatial growth regressions: Model specification, estimation and interpretation. Spatial Economic Analysis, 3(3):275-304.

Leung, Y., Mei, C. L., and Zhang, W. X. (2000a). Statistical tests for spatial nonstationarity based on the geographically weighted regression model. Environment and Planning A, $32(1): 9-32$.

Leung, Y., Mei, C. L., and Zhang, W. X. (2000b). Testing for spatial autocorrelation among the residuals of the geographically weighted regression. Environment and Planning A, $32(5): 871-890$.

McQuarrie, A. D. R.; Tsai, C.-L. (1998). Regression and time series model selection. World Scientific.

Ord, K. (1975). Estimation Methods for Models of Spatial Interaction. Journal of the American Statistical Association, 70(349):120-126.

Paez, A., Uchida, T., and Miyamoto, K. (2002a). A general framework for estimation and inference of geographically weighted regression models: 1. Location-specific kernel bandwidths and a test for locational heterogeneity. Environment and Planning A, 34(4):733754 .

Paez, A., Uchida, T., and Miyamoto, K. (2002b). A general framework for estimation and inference of geographically weighted regression models: 2. Spatial association and model specification tests. Environment and Planning A, 34(5):883-904.

Pötscher, B. M. and Prucha, I. R. (1997). Dynamic Nonlinear Econometric Models. Springer Berlin Heidelberg, Berlin, Heidelberg.

Rao, R. R. (1962). Relations between Weak and Uniform Convergence of Measures with Applications. The Annals of Mathematical Statistics, 33(2):659-680. 
Sin, C.-Y. and White, H. (1996). Information criteria for selecting possibly misspecified parametric models. Journal of Econometrics, 71(1):207-225.

Straumann, D. and Mikosch, T. (2006). Quasi-maximum-likelihood estimation in conditionally heteroeskedastic time series: A stochastic recurrence equations approach. The Annals of Statistics, 34(5):2449-2495.

Su, S., Xiao, R., and Zhang, Y. (2012). Multi-scale analysis of spatially varying relationships between agricultural landscape patterns and urbanization using geographically weighted regression. Applied Geography, 32(2):360-375.

Teräsvirta, T. (1994). Specification, Estimation and Evaluation of Smooth Transition Autoregressive Models. Journal of the American Statistical Association, 89(425):208-218.

Terasvirta, T. and Anderson, H. M. (1992). Teräsvirta_Anderson_1992_Characterizing Nonlinearities in Business Cycles Using Smooth Transition Autoregressive Models. Journal of Applied Econometrics, 7(S):S119-36.

Tong, H. (1978). On a Threshold Model. In Pattern Recognition and Signal Processing, number January 1978, pages 575-586.

Tong, H. (2015). Threshold models in time series analysis-Some reflections. Journal of Econometrics, 189(2):485-491.

Tsay, R. S. (1989). Testing and Modeling Threshold Autoregressive Processes. Journal of the American Statistical Association, 84(405):231-240.

van der Vaart, A. W. (2000). Asymptotic Statistics (Cambridge Series in Statistical and Probabilistic Mathematics). Cambridge University Press.

Wheeler, D. and Tiefelsdorf, M. (2005). Multicollinearity and correlation among local regression coefficients in geographically weighted regression. Journal of Geographical Systems, 7(2):161-187.

Wheeler, D. C. (2007). Diagnostic tools and a remedial method for collinearity in geographically weighted regression. Environment and Planning A, 39(10):2464-2481.

White, H. (1994a). Estimation, inference, and specification analysis. Cambridge University Press.

White, H. (1994b). Estimation, Inference and Specification Analysis. Cambridge Books. Cambridge University Press. 


\section{A Apendix}

\section{A.1 Proof of Theorem 1}

Proof. Note first that $\ell_{T}(\theta):=(1 / T) \sum_{t=1}^{T} \ell_{t}(\theta)$ is a.s. continuous (a.s.c.) in $\theta \in \Theta$ through continuity (c.) of each term $\ell_{t}(\theta)=\ln \operatorname{det} H\left(\theta^{\rho} ; Z_{t}\right)+\ln p_{\varepsilon}\left(H\left(\theta^{\rho} ; Z_{t}\right) Y_{t}-\phi Y_{t-1}-X_{t} \beta-\right.$ $\mu \varepsilon_{t-1}, \Sigma ; \lambda$ ). Together with the compactness of $\Theta$ (Assumption 1) this implies by Weierstrass' theorem that the arg max set is non-empty a.s. and hence that $\hat{\theta}_{T}$ exists a.s. $\forall T \in \mathbb{N}$. Note by a similar argument that $\ell_{T}(\theta)$ is continuous in $\left(Y_{t}, X_{t}\right) \forall \theta \in \Theta$ and hence measurable w.r.t. the product Borel $\sigma$-algebra $\mathfrak{B}(\mathcal{Y}) \otimes \mathfrak{B}(\mathcal{X})$ that are, in turn, measurable maps w.r.t. $\mathcal{F}$ by Proposition 4.1.7 in Dudley (2002). ${ }^{10}$ Finally, the measurability of $\hat{\theta}_{T}$ follows from (Foland, 2009, p.24) and (White, 1994b, Theorem 2.11) or (Gallant and White, 1988, Lemma 2.1, Theorem 2.2). ${ }^{11}$

\section{A.2 Proof of Theorem 2}

Proof. Following a classical consistency argument (found e.g. in (White, 1994b, Theorem 3.4) or Theorem 3.3 in Gallant and White (1988)) we obtain $\hat{\theta}_{T} \stackrel{\text { a.s. }}{\rightarrow} \theta_{0}$ from the uniform convergence of the criterion function

$$
\sup _{\theta \in \Theta}\left|\ell_{T}(\theta)-\ell_{\infty}(\theta)\right| \stackrel{\text { a.s. }}{\rightarrow} 0 \forall f_{1} \in \mathcal{F} \text { as } T \rightarrow \infty
$$

and the identifiable uniqueness of the maximizer $\theta_{0} \in \Theta$ introduced in White (1994b),

$$
\sup _{\theta:\left\|\theta-\theta_{0}\right\|>\epsilon} \ell_{\infty}(\theta)<\ell_{\infty}\left(\theta_{0}\right) \forall \epsilon>0
$$

The uniform convergence is obtained by norm sub-additivity, ${ }^{12}$

$$
\sup _{\theta \in \Theta}\left|\ell_{T}(\theta)-\ell_{\infty}(\theta)\right| \leq \sup _{\theta \in \Theta}\left|\ell_{T}(\theta)-\ell_{T}(\theta)\right|+\sup _{\theta \in \Theta}\left|\ell_{T}(\theta)-\ell_{\infty}(\theta)\right|
$$

and then for the first term showing that

$$
\sup _{\theta \in \Theta}\left|\ell_{T}(\theta)-\ell_{T}(\theta)\right| \stackrel{\text { a.s. }}{\rightarrow} 0 \text { as } T \rightarrow \infty
$$

\footnotetext{
${ }^{10}$ Dudley's proposition states that the Borel $\sigma$-algebra $\mathfrak{B}(\mathbb{A} \times \mathbb{B})$ generated by the Tychonoff's product topology $\mathcal{T}_{\mathbb{A} \times \mathbb{B}}$ on the space $\mathbb{A} \times \mathbb{B}$ includes the product $\sigma$-algebra $\mathfrak{B}(\mathbb{A}) \otimes \mathbb{B}(\mathbb{B})$.

${ }^{11}$ The reference of Foland (2009) is used here to establish that a map into a product space is measurable if and only if its projections are measurable.

${ }^{12} \ell_{T}(\theta)$ denotes the limit as $T \rightarrow \infty$ of $\ell_{T}(\theta)$ defined as $\ell_{T}(\theta):=(1 / T) \sum_{t=1}^{T} \ell_{t}(\theta)$ with $\ell_{t}(\theta)$ defined in (9).
} 
and for the second term applying the ergodic theorem for separable Banach spaces in Rao (1962), as in (Straumann and Mikosch, 2006, Theorem 2.7), to the sequence $\left\{\ell_{T}(\cdot)\right\}$ with elements taking values in $\mathbb{C}(\Theta, \mathbb{R})$ so that $\sup _{\theta \in \Theta}\left|\ell_{T}(\theta)-\ell_{\infty}(\theta)\right|$ where $\ell_{\infty}(\theta)=\mathbb{E} \ell_{t}(\theta) \forall \theta \in$ $\Theta$.

The ULLN $\sup _{\theta \in \Theta}\left|\ell_{T}(\theta)-\mathbb{E} \ell_{t}(\theta)\right| \stackrel{\text { a.s. }}{\rightarrow} 0$ as $T \rightarrow \infty$ follows, under a moment bound $\mathbb{E} \sup _{\theta \in \Theta}\left|\ell_{t}(\theta)\right|<\infty$, by the SE nature of $\left\{\ell_{T}\right\}_{t \in \mathbb{Z}}$ which is implied by continuity of $\ell$ on the SE sequence $\left\{\left(Y_{t}, X_{t}\right)\right\}_{t \in \mathbb{Z}}$ (Assumption 2) and Proposition 4.3 in Krengel (1985). The moment bound $\mathbb{E} \sup _{\theta \in \Theta}\left|\ell_{t}(\theta)\right|<\infty$ follows immediately from Assumption 3 since

$$
\mathbb{E} \sup _{\theta \in \Theta}\left|\ell_{t}(\theta)\right| \leq \mathbb{E} \sup _{\theta \in \Theta}\left|Q\left(\theta^{\rho} ; Z_{t}\right)\right|+|A(\theta)|+\frac{1}{2}(\lambda+n) \mathbb{E} \sup _{\theta \in \Theta}\left|F\left(\theta, Y_{t}, X_{t}, Z_{t}\right)\right|<\infty
$$

Finally, the identifiable uniqueness (see e.g. White (1994b)) of $\theta_{0} \in \Theta$ in (13) follows from the assumed uniqueness (Assumption 3), the compactness of $\Theta$, and the continuity of the limit $\mathbb{E} \ell_{t}(\theta)$ in $\theta \in \Theta$ which is implied by the continuity of $\ell_{T}$ in $\theta \in \Theta \forall T \in \mathbb{N}$ and the uniform convergence in (12).

\section{A.3 Proof of Theorem 3}

Proof. We obtain the asymptotic Gaussianity of the MLE from (i) the strong consistency of $\hat{\theta}_{T} \stackrel{\text { a.s. }}{\rightarrow} \theta_{0} \in \operatorname{int}(\Theta)$; (ii) the a.s. twice continuous differentiability of $\ell_{T}(\theta)$ in $\theta \in \Theta$; (iii) the asymptotic normality of the score

$$
\sqrt{T} \ell_{T}^{\prime}\left(\theta_{0}\right) \stackrel{d}{\rightarrow} N\left(0, \mathcal{J}\left(\theta_{0}\right)\right), \quad \mathcal{J}\left(\theta_{0}\right)=\mathbb{E}\left(\ell_{t}^{\prime}\left(\theta_{0}\right) \ell_{t}^{\prime}\left(\theta_{0}\right)^{\top}\right)
$$

(iv) the uniform convergence of the likelihood's second derivative,

$$
\sup _{\theta \in \Theta}\left\|\ell_{T}^{\prime \prime}(\theta)-\ell_{\infty}^{\prime \prime}(\theta)\right\| \stackrel{a . s .}{\rightarrow} 0
$$

and finally, (v) the non-singularity of the limit $\ell_{\infty}^{\prime \prime}(\theta)=\mathbb{E} \ell_{t}^{\prime \prime}(\theta)=\mathcal{I}(\theta)$. See e.g. in (White, 1994b, Theorem 6.2)) for further details.

The consistency condition $\hat{\theta}_{T} \stackrel{\text { a.s. }}{\rightarrow} \theta_{0} \in \operatorname{int}(\Theta)$ in (i) follows by Theorem 2 and the additional assumption that $\theta_{0} \in \operatorname{int}(\Theta)$.

The smoothness condition in (ii) is trivially satisfied for the student's- $t$ density.

The asymptotic normality of the score in (17) follows by Theorem 18.10[iv] in van der Vaart (2000) by an application of the CLT for SE martingales in Billingsley (1961) to obtain

$$
\sqrt{T} \ell_{T}^{\prime}\left(\theta_{0}\right) \stackrel{d}{\rightarrow} N\left(0, \mathcal{J}\left(\theta_{0}\right)\right) \text { as } T \rightarrow \infty
$$


where $\mathcal{J}\left(\theta_{0}\right)=\mathbb{E}\left(\ell_{t}^{\prime}\left(\theta_{0}\right) \ell_{t}^{\prime}\left(\theta_{0}\right)^{\top}\right)<\infty$. The SE nature of $\left\{\ell_{T}^{\prime}\left(\theta_{0}\right)\right\}_{t \in \mathbb{Z}}$ follows by continuity of $\ell^{\prime}$ on the SE sequence $\left\{\left(Y_{t}, X_{t}\right)\right\}_{t \in \mathbb{Z}}$; see Proposition 4.3 in Krengel (1985). Assumption 5 imposes the mds nature of $\left\{\ell_{T}^{\prime}\left(\theta_{0}\right)\right\}_{t \in \mathbb{Z}}$. The finite (co)variances follow from the first two moments bounds of Assumption 6 .

The uniform convergence in (iv)is obtained under the moment bound $\mathbb{E} \sup _{\theta \in \Theta}\left\|\ell_{t}^{\prime \prime}(\theta)\right\|<$ $\infty$ and by the SE nature of $\left\{\ell_{T}^{\prime \prime}\right\}_{t \in \mathbb{Z}}$. The moment bound is ensured by Assumption 6 . The SE nature is implied by continuity of $\ell^{\prime \prime}$ on the SE sequence $\left\{Y_{t}, X_{t}\right\}_{t \in \mathbb{Z}}$.

Finally, the non-singularity of the limit $\ell_{\infty}^{\prime \prime}(\theta)=\mathbb{E} \ell_{t}^{\prime \prime}(\theta)=\mathcal{I}(\theta)$ in $(\mathrm{v})$ is implied by the uniqueness of $\theta_{0}$ as a maximum of $\ell_{\infty}^{\prime \prime}(\theta)$ in $\Theta$. 


\section{Supplementary Appendix}

\section{Smooth Transition Spatial Autoregressive Models for Cross-sectional Time Series}

Bo Pieter Johannes Andrée, Francisco Blasques, Eric Koomen

\section{Additional Results}

As a potential DGP the ST-SAR is capable of producing random data with interesting nonlinear dynamics over time and space. Because of the multivariate nature of the model, a proof for strict stationarity and ergodicity based on Lipschitz properties is unavailable, see for example Comte and Lieberman (2003) for a discussion in the context of the multivartiate GARCH. ${ }^{13}$. Therefore, results regarding strict stationarity and ergodicity of time-varying spatial parameter models provided by Blasques et al. (2016) do not hold for the ST-SAR.

While the ST-SAR dynamics may be complex, under appropriate regularity conditions, the model can still be shown to generate data that is geometrically ergodic. We focus on the endogenous case where $Z_{t}=Y_{t}$ and $\beta=0$ as this is naturally the most interesting case for a study on stability. Let $\theta$ denote the vector of parameters of our ST-SAR $\theta:=(\delta, \gamma, \alpha, \varphi, \kappa, \phi)^{\prime}$ and $\theta_{0}$ denote the vector of the true parameters of the endogenous ST-SAR. Under correct specification, there exists $\theta_{0} \in \Theta$, such that an observed $T$-period data sequence $\left\{Y_{t}(\omega)\right\}_{t=1}^{T}$ is a subset of a realized path of the $n$-variate stochastic sequence $Y(\omega):=\left\{Y_{t}(\omega)\right\}_{t \in \mathbb{Z}}$, for some event $\omega$ in the event space $\Omega$ with $Y_{t}(\omega) \in \mathcal{Y} \subset \mathbb{R}^{n} \forall(\omega, t) \in \Omega \times \mathbb{Z}$ living on the space $\left(\mathcal{Y}_{\infty}, \mathfrak{B}\left(\mathcal{Y}_{\infty}\right), P_{0}^{y}\right)$. $P_{0}^{y}$ here denotes the true probability measure, such that we are concerned with a sequence $Y(\omega)$ generated by the model. Below we let $\|\cdot\|_{\infty}$ denote the sup norm on $\mathbb{R}^{n}$ and $\|\cdot\|$ denote any matrix norm. For readability, we first present the results and let the proofs follow at the end of the section.

Lemma 1. Let $A$ be an arbitrary finite-dimensional matrix. For an induced matrix norm $\|A\|<1$ the following inequality is implied:

$$
(1+\|A\|)^{-1} \leq\left\|\left(I_{n}-A\right)^{-1}\right\| \leq(1-\|A\|)^{-1},
$$

\footnotetext{
${ }^{13} \mathrm{~A}$ model $Y_{t}=F\left(Y_{t-1}, \varepsilon_{t}\right)$ is Lipschitz if $\|F(x, \varepsilon)-F(y, \varepsilon)\| \leq \alpha(\varepsilon)\|x-y\|$ for a positive valued function $\alpha$ with $\mathbb{E}\left(\alpha\left(\varepsilon_{t}\right)^{m}\right)<1$ and $\mathbb{E}\left(\left\|F\left(0, \mid \varepsilon_{t}\right)\right\|^{m}\right)<\infty$ for some real $m \geq 1$.
} 
with $0<(1+\|A\|)^{-1}$ and $(1-\|A\|)^{-1}<\infty$. By the finite-dimensionality we can also write

$$
0<c \leq\left\|\left(I_{n}-A\right)^{-1}\right\|_{\infty} \leq C<\infty
$$

for some positive constants $c$ and $C$.

For a matrix $H$ defined by $H=\left(I_{n}-A\right)$, Lemma 1 provides existence, non-negativity, and boundedness of the inverse $H^{-1}$ for finite dimensional $H$. This is useful since throughout our theory as we always work with a fixed $n$ and let only $T$ tend to infinity.

Lemma 2. Let $A$ be an arbitrary matrix with eigenvalues $\omega_{1}, \ldots, \omega_{n} \in \mathbb{C}^{n \times n}$, real or complex, and $r(A)=\max \left\{\left|\omega_{1}\right|, \ldots,\left|\omega_{n}\right|\right\}$ be its spectral radius. If $r(A)<1$ there exists $\|A\|<1$ for some induced matrix norm.

Lemma 2 allows the condition $\|A\|<1$ in Lemma 1 to be replaced by $r(A)<1$ if no suitable norm can be found. In what follows we will continue stating $\|\cdot\|$, but remind the reader that in practice one may focus on sample estimates of $r(\cdot)$ as a rule of thumb.

Lemma 3. For any $H^{-1} \in \mathbb{R}^{n \times n}$ defined as $H^{-1}=\left(I_{n}-A\right)^{-1}$ with $n<\infty$ and $r(A)<1$, we have that the following is implied

$$
\begin{aligned}
& i \operatorname{det}\left(H^{-1}\right)>0, \\
& \text { ii } 0<\log \tau\left(H^{-1}\right)^{n} \leq \log \operatorname{det}\left(H^{-1}\right) \leq \log r\left(H^{-1}\right)^{n}<\infty, \\
& \text { iii }\left|\log \operatorname{det}\left(H^{-1}\right)\right|<\infty
\end{aligned}
$$

Claim iii in Lemma 3 is particularly useful in establishing that Assumption 3 holds under correct specification.

The following Proposition now establishes the $\psi$-irreducibility of T-chains generated by the ST-SAR.

Proposition 1. Let $\epsilon_{t}$ have full support, and $\sup _{Y \in \mathbb{R}^{n}}\left\|\rho\left(\theta_{0}^{\rho} ; Y\right)\right\|<1$. Then $\left\{Y_{t}\right\}_{t \in \mathbb{N}}$ is an aperiodic, $\psi$-irreducible, T-Chain.

Proposition 2. Let the conditions of Proposition 1 hold. Assume further that $\left\|\epsilon_{t}\right\|^{r}<\infty$ for some $r>0$, and $\lim _{\|Y\| \rightarrow \infty} H(Y)^{-1}=H_{\infty} \in \mathbb{R}^{p \times p}$ with $\left\|H_{\infty}\right\||\phi|<1$. Then $\left\{Y_{t}\right\}_{t \in \mathbb{N}}$ is geometrically ergodic.

Under additional regularity conditions, the consistency result is also available for the case of a correctly-specified ST-SAR model. Corollary 1 makes use of Proposition 2 to obtain the consistency of the MLE $\hat{\theta}_{T}$ with respect to $\theta_{0}$. Note that, this time, the parameter $\theta_{0}$ does indeed correspond to the true parameter that defines the true distribution of the data. 
Corollary 1 (Consistency under Correct Specification). Let $\left\{Y_{t}\right\}_{t \in \mathbb{Z}}$ be generated by the $S T-S A R$ model Equation (4) under some $\theta_{0} \in \Theta$. Furthermore, let assumptions 1 and 3 hold, and $\Theta$ be such that $\sup _{Y \in \mathbb{R}^{n}}\left\|\rho\left(\theta_{0}^{\rho} ; Y\right)\right\|<1$ and $\Sigma$ is positive definite for every $\theta \in \Theta$. Then the MLE satisfies $\hat{\theta}_{T} \stackrel{\text { a.s. }}{\longrightarrow} \theta_{0}$ as $T \rightarrow \infty$.

\section{Proofs for additional results}

\section{Proof of Lemma 1}

Proof. The result follows by taking norms in $I_{n}=\left(I_{n}-A\right)\left(I_{n}-A\right)^{-1}$, which gives ${ }^{14}$

$$
\begin{aligned}
1 & \leq\left\|I_{n}-A\right\|\left\|\left(I_{n}-A\right)^{-1}\right\|, \\
& \leq(1+\|A\|)\left\|\left(I_{n}-A\right)^{-1}\right\| .
\end{aligned}
$$

Multiplying by $\left\|\left(I_{n}-A\right)^{-1}\right\|^{-1}$ gives

$$
\left\|\left(I_{n}-A\right)^{-1}\right\|^{-1} \leq\left\|I_{n}-A\right\| \leq(1+\|A\|),
$$

thus

$$
\begin{gathered}
\left(\left\|\left(I_{n}-A\right)^{-1}\right\|\right)^{-1} \leq(1+\|A\|), \\
(1+\|A\|)^{-1} \leq\left\|\left(I_{n}-A\right)^{-1}\right\|,
\end{gathered}
$$

providing the first inequality.

The second inequality follows by application of the binomial inverse theorem:

$$
(C+U B V)^{-1}=C^{-1}-C^{-1} U B\left(B+B V C^{-1} U B\right)^{-1} B V C^{-1} .
$$

Setting $C=U=V=I_{n}$ gives the simpler identity

$$
(C+B)^{-1}=C^{-1}-C^{-1} B\left(B+B C^{-1} B\right)^{-1} B C^{-1} .
$$

Applying the straightforward identity $(B C)^{-1}=B^{-1} C^{-1}$, we get

$$
(C+B)^{-1}=C^{-1}-C^{-1}\left(I_{n}+B C^{-1}\right)^{-1} B C^{-1} .
$$

Now setting $C=I_{n}$ and $B=-A$ we get

$$
\left(I_{n}-A\right)^{-1}=I_{n}+\left(I_{n}-A\right)^{-1} A .
$$

Taking norms gives us the following inequality:

$$
\left\|\left(I_{n}-A\right)^{-1}\right\| \leq 1+\left\|\left(I_{n}-A\right)^{-1}\right\|\|A\|,
$$

which we can re-arrange to obtain the second inequality

\footnotetext{
${ }^{14}$ The result is similar to Proposition 6.4.1. in Lange (1999), but reworked here because both the proof and the final result are partial.
} 


$$
\begin{gathered}
\left\|\left(I_{n}-A\right)^{-1}\right\| \leq 1+\left\|\left(I_{n}-A\right)^{-1}\right\|\|A\|, \\
\left\|\left(I_{n}-A\right)^{-1}\right\| \leq 1+\|A\|\left\|\left(I_{n}-A\right)^{-1}\right\|, \\
\left\|\left(I_{n}-A\right)^{-1}\right\|-\|A\|\left\|\left(I_{n}-A\right)^{-1}\right\| \leq 1, \\
1-\|A\| \leq\left\|\left(I_{n}-A\right)^{-1}\right\|^{-1}, \\
(1-\|A\|)^{-1} \geq\left\|\left(I_{n}-A\right)^{-1}\right\|, \\
\left\|\left(I_{n}-A\right)^{-1}\right\| \leq(1-\|A\|)^{-1} .
\end{gathered}
$$

Finiteness of $\left\|\left(I_{n}-A\right)^{-1}\right\|$ follows trivially from

$$
(1-\|A\|)^{-1}<\infty \text {. since }\|A\|<1 .
$$

Non-negativity of $\left(I_{n}-A\right)^{-1}$ follows by noting that all its eigenvalues are non-zero. The minimum eigenvalue of a non-singular matrix is equal to the inverse of the spectral radius of the inverse matrix, thus in this case $\tau\left(\left(I_{n}-A\right)^{-1}\right)=r(I-A)^{-1}$. Having just established that $(1+\|A\|)^{-1} \leq\left\|\left(I_{n}-A\right)^{-1}\right\| \leq(1-\|A\|)^{-1}$ it follows trivially that like-wise

$$
(1+\|A\|) \geq\left\|\left(I_{n}-A\right)\right\| \geq(1-\|A\|),
$$

which delivers the upper bounds of $r\left(I_{n}-A\right)$ by noting that $r\left(I_{n}-A\right) \leq\left\|I_{n}-A\right\|$, hence $r(I-A)^{-1}>0$, and equally so $\tau\left(\left(I_{n}-A\right)^{-1}\right)>0$.

Finally, by noting that any two norms in finite dimension $n<\infty$ are always within a constant factor of one another, such that we can write for some real numbers $0<c_{1} \leq c_{1} \leq$ $c_{2}$ the inequality

$$
c_{1}\left\|\left(I_{n}-A\right)^{-1}\right\|_{\infty} \leq\left\|\left(I_{n}-A\right)^{-1}\right\| \leq c_{2}\left\|\left(I_{n}-A\right)^{-1}\right\|_{\infty},
$$

proves the second claim by setting $c=c_{1}\left\|\left(I_{n}-A\right)^{-1}\right\|_{\infty}$ and $C=c_{2}\left\|\left(I_{n}-A\right)^{-1}\right\|_{\infty}$.

\section{Proof of Lemma 2}

Proof. This follows from by noting that for any matrix $A$ and any positive number $\varepsilon>0$, there exists an induced matrix norm $\|A\|$ such that

$$
r(A) \leq\|A\|<r(A)+\varepsilon .
$$

See for Proposition 6.3.2. Lange (1999). Trivially,

$$
r(A)<1 \Longrightarrow 1-r(A)>0 .
$$

Choose $\varepsilon=1-r(A)$, the proof is complete by noting that we can now write 


$$
\begin{aligned}
& r(A) \leq\|A\|<r(A)+1-r(A) \\
& r(A) \leq\|A\|<1
\end{aligned}
$$

\section{A.3.1 Proof of Lemma 3}

Proof. The proof of all three claims starts by noting that by definition

$$
\operatorname{det}\left(H^{-1}\right)=\left(\Pi_{i=1}^{k} \omega_{i}\right)\left(\Pi_{i=k+1}^{p} \omega_{i} \bar{\omega}_{i}\right)=\left(\Pi_{i=1}^{k} \omega_{i}\right)\left(\Pi_{i=k+1}^{p}\left|\omega_{i}\right|^{2}\right),
$$

with $\omega_{1}, \ldots, \omega_{n} \in \mathbb{C}^{n \times n}$, real or complex, as the eigenvalues of $H^{-1}$. Hence the first claim follows by showing that

$$
\left(\Pi_{i=1}^{k} \omega_{i}\right)\left(\Pi_{i=k+1}^{p}\left|\omega_{i}\right|^{2}\right)>0
$$

Thus we need to show that $\omega_{i}>0 \forall i \in n$, since then $\left(\left|\omega_{i}\right|^{2}\right)^{p-k}>0$, and $\omega_{i}^{n-p}>0$, hence the left side of the second equality is strictly positive.

Note that Lemma 1 and Lemma 2 deliver the following inequality under assumptions of Lemma 3,

$$
(1+\|A\|)^{-1} \leq\left\|\left(I_{n}-A\right)^{-1}\right\| \leq(1-\|A\|)^{-1},
$$

which we can also write as

$$
(1-\|A\|) \leq\left\|\left(I_{n}-A\right)\right\| \leq(1+\|A\|) .
$$

The desirable result follows by proving that $\tau\left(H^{-1}\right)>0$, where $\tau\left(H^{-1}\right)=\tau\left(\left(I_{n}-A\right)^{-1}\right)=$ $\min \left\{\left|\omega_{1}\right|, \ldots,\left|\omega_{n}\right|\right\}$. Applying the useful identity $\tau(A)=\left(r\left(A^{-1}\right)\right)^{-1}$, we have

$$
\tau\left(H^{-1}\right)=(r(H))^{-1},
$$

hence showing that $\tau\left(H^{-1}\right)>0$ equals showing that $(r(H))^{-1}>0$, which follows from $r(H)<\infty$. Using the general inequality $r(H) \leq\|H\|$ we can write $r(H) \leq\left\|\left(I_{n}-A\right)\right\| \leq$ $(1+\|A\|)$ thus proving $\tau\left(H^{-1}\right)>0$. Using the definition of $\operatorname{det}\left(H^{-1}\right)$, and the bounds of $H^{-1}$ we obtain the range of the determinant by allowing the finite number of $n$ eigenvalues to be either strictly minima or maxima

$$
0<\left(\tau\left(H^{-1}\right)^{n} \leq \operatorname{det}\left(H^{-1}\right) \leq r\left(H^{-1}\right)^{n}<\infty .\right.
$$

The second claim follows easily now by taking logs and applying Jensen's inequality.

Finally, the third claim follows by noting that $0<\operatorname{det}\left(H^{-1}\right)$ implies that the $\log$ is defined, hence its absolute value is finite. 


\section{Proof of Proposition 1}

Proof. The result follows by Theorem 2.2 and Example 2.1 in Cline and $\mathrm{Pu}$ (1998). In particular, we note first that Lemma 1 provides the uniform bound of $H(Y)^{-1}$ by noting that if $\sup _{Y \in \mathbb{R}^{n}}\left\|\rho\left(\theta_{0}^{\rho} ; Y\right) \circ W\right\|<1$ we have

$$
0<\bar{h} \leq \sup _{Y \in \mathbb{R}^{n}}\left\|H(Y)^{-1}\right\| \leq \bar{H}<\infty
$$

with $\bar{h}=\left(1+\sup _{Y \in \mathbb{R}^{n}}\left\|\rho\left(\theta_{0}^{\rho} ; Y\right) \circ W\right\|\right)^{-1}$ and $\bar{H}=\left(1-\sup _{Y \in \mathbb{R}^{n}}\left\|\rho\left(\theta_{0}^{\rho} ; Y\right) \circ W\right\|\right)^{-1}$. Having just established that $\sup _{Y \in \mathbb{R}^{n}}\left\|H(Y)^{-1}\right\|$ is bounded away from zero by some constant $\bar{h}$ and from infinity by some constant $\bar{H}<\infty$, and that $H^{-1}(Y)$ is invertible, we can now verify that the assumptions in Theorem 2.2 and Example 2.1 in Cline and Pu (1998) hold. First we note that $H(Y)$ and $H(Y)^{-1}$ are both trivially locally bounded, and that $\epsilon_{t}$ has full support. Finally, we note that $H(Y)^{-1} Y \phi$ is also locally bounded since

$$
\sup _{\|Y\| \leq M}\left\|H(Y)^{-1} Y \phi\right\| \leq \sup _{Y}\left\|H(Y)^{-1}\right\||\phi| \sup _{\|Y\| \leq M}\|Y\| \leq B M \phi<\infty \quad \forall M>0 .
$$

\section{Proof of Proposition 2}

Proof. We recall that $\sup _{Y i n \mathbb{R}^{n}}\left\|H(Y)^{-1}\right\| \leq B<\infty$ under the assumptions of Proposition 1. Next we obtain the desired result from Theorem 3.1 of Cline and $\mathrm{Pu}$ (1999). First, we note that $H(Y)^{-1} Y$ is trivially unbounded in $\mathbb{R}^{n}$. Second, we have that $H(Y)^{-1} Y /(1+\|Y\|)$ is bounded in $\mathbb{R}^{n}$ since

$$
\left\|H(Y)^{-1} Y \phi /(1+\|Y\|)\right\| \leq\left\|H(Y)^{-1}\right\|\|Y\||\phi| /(1+\|Y\|) \leq B\|Y\||\phi| /(1+\|Y\|) \leq B|\phi| .
$$

Next, we note that

$$
\sup _{\|Y\| \leq M} \mathbb{E}\left\|H(Y)^{-1} \epsilon_{t}\right\|^{r} \leq \sup _{\|Y\| \in \mathbb{R}^{n}}\left\|H(Y)^{-1}\right\| \mathbb{E}\left\|\epsilon_{t}\right\|^{r} \leq B \mathbb{E}\left\|\epsilon_{t}\right\|^{r}<\infty \quad \text { for every } M>0 .
$$

Additionally, it holds trivially true that

$$
\lim _{\|Y\| \rightarrow \infty} \mathbb{E} \frac{\left\|H(Y)^{-1} \epsilon_{t}\right\|^{r}}{\|Y\|^{r}} \leq \lim _{\|Y\| \rightarrow \infty} \frac{B\left\|\epsilon_{t}\right\|^{r}}{\|Y\|^{r}} \rightarrow 0 .
$$


Furthermore, it holds true that

$\lim _{\substack{\|H(Y)-1 Y \phi\| \rightarrow \infty \\\left\|Y-Y^{\prime}\right\| /\|Y\| \rightarrow 0}}\left\|\frac{H(Y)^{-1} Y \phi}{1+\|Y\|}-\frac{H\left(Y^{\prime}\right)^{-1} Y^{\prime} \phi}{1+\left\|Y^{\prime}\right\|}\right\|=\lim _{\substack{\|Y\| \rightarrow \infty,\left\|Y^{\prime}\right\| \rightarrow \infty \\\left\|Y-Y^{\prime}\right\| /\|Y\| \rightarrow 0}}\left\|\frac{H(Y)^{-1} Y \phi}{1+\|Y\|}-\frac{H\left(Y^{\prime}\right)^{-1} Y^{\prime} \phi}{1+\left\|Y^{\prime}\right\|}\right\|$

since $H(Y)^{-1}$ is uniformly bounded in $Y$, and hence,

$$
\begin{gathered}
\left\|H(Y)^{-1} Y \phi\right\| \rightarrow \infty \quad \Leftrightarrow \quad\|Y\| \rightarrow \infty, \\
\text { and }\left\{\|Y\| \rightarrow \infty \wedge\left\|Y-Y^{\prime}\right\| /\|Y\| \rightarrow 0\right\} \quad \Leftrightarrow \quad\left\|Y^{\prime}\right\| \rightarrow \infty .
\end{gathered}
$$

As a result

$$
\lim _{\substack{\|Y\| \rightarrow \infty,\left\|Y^{\prime}\right\| \rightarrow \infty \\\left\|Y-Y^{\prime}\right\| /\|Y\| \rightarrow 0}}\left\|\frac{H(Y)^{-1} Y \phi}{1+\|Y\|}-\frac{H\left(Y^{\prime}\right)^{-1} Y^{\prime} \phi}{1+\left\|Y^{\prime}\right\|}\right\|=\left\|H_{\infty} \phi-H_{\infty} \phi\right\|=0 .
$$

Finally, we also have

$$
\limsup _{\|Y\| \rightarrow \infty} \frac{\left\|H(Y)^{-1} Y \phi\right\|}{\|Y\|} \leq \limsup _{\|Y\| \rightarrow \infty} \frac{\left\|H(Y)^{-1}\right\|\|Y\||\phi|}{\|Y\|}=\left\|H_{\infty}\right\||\phi|<1 .
$$




\section{Additional Monte Carlo results}

Table 7: Selection frequencies for data generated from the ST-SAR.

\begin{tabular}{|c|c|c|c|c|c|c|c|c|c|c|}
\hline \multicolumn{2}{|c|}{ ST-SAR DGP } & \multicolumn{3}{|c|}{$\begin{array}{l}\text { ST-SAR } 1 \\
\text { vs. SAR }\end{array}$} & \multicolumn{3}{|c|}{$\begin{array}{l}\text { ST-SAR } 2 \\
\text { vs. SAR }\end{array}$} & \multicolumn{3}{|c|}{$\begin{array}{c}\text { ST-SAR } 2 \\
\text { vs. ST-SAR } 1\end{array}$} \\
\hline & & AIC & $\mathrm{AICc}$ & mAIC & $\mathrm{AIC}$ & $\mathrm{AICc}$ & mAIC & $\mathrm{AIC}$ & $\mathrm{AICc}$ & $\mathrm{mAIC}$ \\
\hline \multirow[t]{5}{*}{$\mathrm{N}=30$} & $\mathrm{~T}=10$ & 47 & 44 & 45 & 45 & 41 & 43 & 42 & 40 & 41 \\
\hline & $\mathrm{T}=25$ & 59 & 58 & 59 & 61 & 60 & 60 & 48 & 48 & 48 \\
\hline & $\mathrm{T}=50$ & 84 & 84 & 84 & 81 & 81 & 81 & 52 & 52 & 52 \\
\hline & $\mathrm{T}=100$ & 97 & 97 & 97 & 96 & 96 & 96 & 62 & 61 & 62 \\
\hline & $\mathrm{T}=250$ & 100 & 100 & 100 & 100 & 100 & 100 & 95 & 95 & 95 \\
\hline \multirow[t]{5}{*}{$\mathrm{N}=40$} & $\mathrm{~T}=10$ & 52 & 51 & 51 & 55 & 53 & 54 & 47 & 45 & 46 \\
\hline & $\mathrm{T}=25$ & 73 & 72 & 72 & 76 & 76 & 76 & 54 & 54 & 54 \\
\hline & $\mathrm{T}=50$ & 93 & 93 & 93 & 91 & 91 & 91 & 54 & 53 & 53 \\
\hline & $\mathrm{T}=100$ & 93 & 93 & 93 & 99 & 99 & 99 & 71 & 71 & 71 \\
\hline & $\mathrm{T}=250$ & 98 & 98 & 98 & 100 & 100 & 100 & 99 & 99 & 99 \\
\hline \multirow[t]{5}{*}{$\mathrm{N}=50$} & $\mathrm{~T}=10$ & 57 & 55 & 56 & 63 & 62 & 63 & 54 & 53 & 54 \\
\hline & $\mathrm{T}=25$ & 83 & 83 & 83 & 80 & 80 & 80 & 51 & 51 & 51 \\
\hline & $\mathrm{T}=50$ & 97 & 97 & 97 & 96 & 96 & 96 & 61 & 61 & 61 \\
\hline & $\mathrm{T}=100$ & 99 & 99 & 99 & 100 & 100 & 100 & 77 & 77 & 77 \\
\hline & $\mathrm{T}=250$ & 100 & 100 & 100 & 100 & 100 & 100 & 99 & 99 & 99 \\
\hline \multirow[t]{5}{*}{$\mathrm{N}=60$} & $\mathrm{~T}=10$ & 65 & 64 & 65 & 63 & 62 & 63 & 49 & 48 & 49 \\
\hline & $\mathrm{T}=25$ & 89 & 89 & 89 & 88 & 88 & 88 & 49 & 49 & 49 \\
\hline & $\mathrm{T}=50$ & 99 & 99 & 99 & 98 & 98 & 98 & 56 & 56 & 56 \\
\hline & $\mathrm{T}=100$ & 99 & 99 & 99 & 100 & 100 & 100 & 80 & 80 & 80 \\
\hline & $\mathrm{T}=250$ & 100 & 100 & 100 & 100 & 100 & 100 & 100 & 100 & 100 \\
\hline
\end{tabular}


Standardized density of estimates for delta

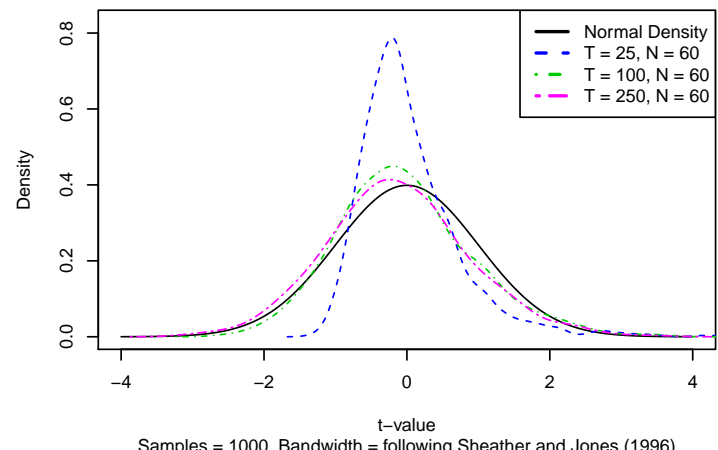

Standardized density of estimates for phi

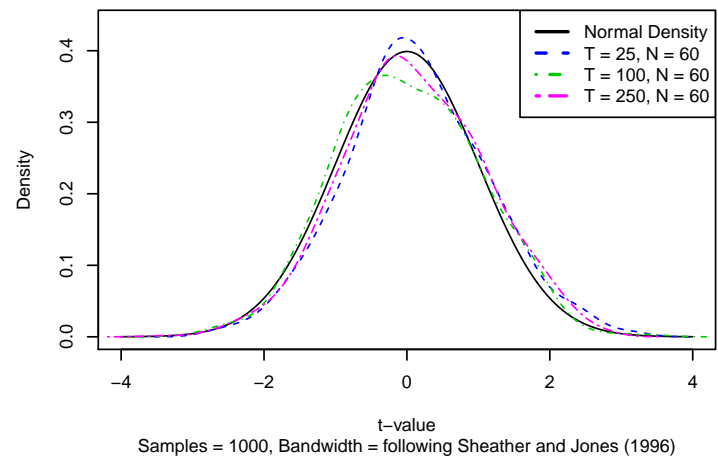

Standardized density of estimates for kappa

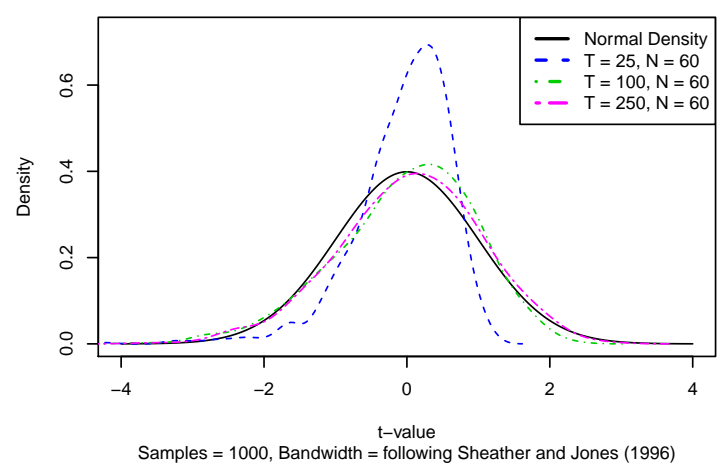

Standardized density of estimates for gamma

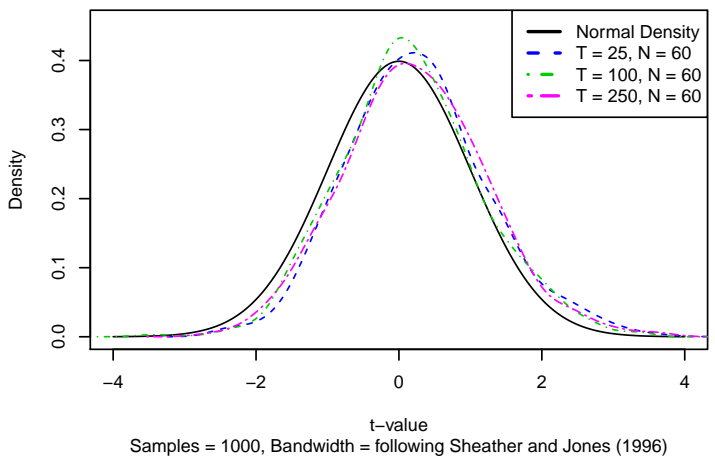

Standardized density of estimates for alpha

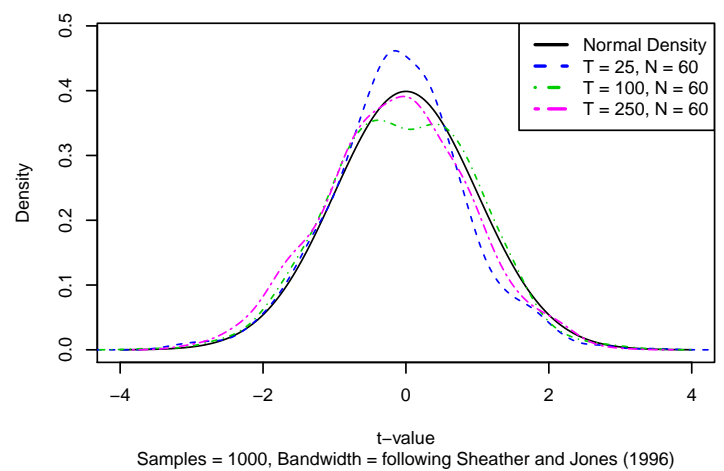

Figure 7: Kernel density estimates of estimated parameters from 1000 simulation replications for $N=60$. 
Table 8: Selection frequencies for (contaminated) data generated from the SAR.

\begin{tabular}{|c|c|c|c|c|c|c|c|}
\hline \multicolumn{2}{|c|}{$\begin{array}{c}\text { SAR (left) } \\
\mathrm{SAR}+\mathrm{AO} \text { (right) }\end{array}$} & \multicolumn{3}{|c|}{$\begin{array}{c}\text { ST-SAR } 2 \\
\text { vs. SAR }\end{array}$} & \multicolumn{3}{|c|}{$\begin{array}{c}\text { ST-SAR } 2 \\
\text { vs. SAR }\end{array}$} \\
\hline & & AIC & $\mathrm{AICc}$ & $\mathrm{mAIC}$ & AIC & AICc & $\mathrm{mAIC}$ \\
\hline \multirow[t]{5}{*}{$\mathrm{N}=30$} & $\mathrm{~T}=10$ & 46 & 44 & 45 & 44 & 42 & 44 \\
\hline & $\mathrm{T}=25$ & 33 & 32 & 32 & 30 & 29 & 30 \\
\hline & $\mathrm{T}=50$ & 27 & 27 & 27 & 26 & 25 & 26 \\
\hline & $\mathrm{T}=100$ & 22 & 22 & 22 & 23 & 23 & 23 \\
\hline & $\mathrm{T}=250$ & 22 & 22 & 22 & 20 & 20 & 20 \\
\hline \multirow[t]{5}{*}{$\mathrm{N}=40$} & $\mathrm{~T}=10$ & 52 & 51 & 51 & 54 & 52 & 53 \\
\hline & $\mathrm{T}=25$ & 32 & 31 & 31 & 33 & 33 & 33 \\
\hline & $\mathrm{T}=50$ & 24 & 24 & 24 & 25 & 25 & 25 \\
\hline & $\mathrm{T}=100$ & 22 & 22 & 22 & 23 & 23 & 23 \\
\hline & $\mathrm{T}=250$ & 23 & 23 & 23 & 18 & 18 & 18 \\
\hline \multirow[t]{5}{*}{$\mathrm{N}=50$} & $\mathrm{~T}=10$ & 41 & 39 & 40 & 43 & 42 & 42 \\
\hline & $\mathrm{T}=25$ & 25 & 25 & 25 & 27 & 26 & 26 \\
\hline & $\mathrm{T}=50$ & 24 & 24 & 24 & 23 & 23 & 23 \\
\hline & $\mathrm{T}=100$ & 20 & 19 & 19 & 19 & 19 & 19 \\
\hline & $\mathrm{T}=250$ & 18 & 18 & 18 & 18 & 18 & 18 \\
\hline \multirow[t]{5}{*}{$\mathrm{N}=60$} & $\mathrm{~T}=10$ & 32 & 31 & 32 & 33 & 32 & 32 \\
\hline & $\mathrm{T}=25$ & 24 & 23 & 24 & 27 & 27 & 27 \\
\hline & $\mathrm{T}=50$ & 24 & 23 & 24 & 26 & 26 & 26 \\
\hline & $\mathrm{T}=100$ & 17 & 17 & 17 & 16 & 16 & 16 \\
\hline & $\mathrm{T}=250$ & 17 & 17 & 17 & 17 & 17 & 17 \\
\hline
\end{tabular}

\section{Additional Figures}

Figure 8: Overview of the study area.

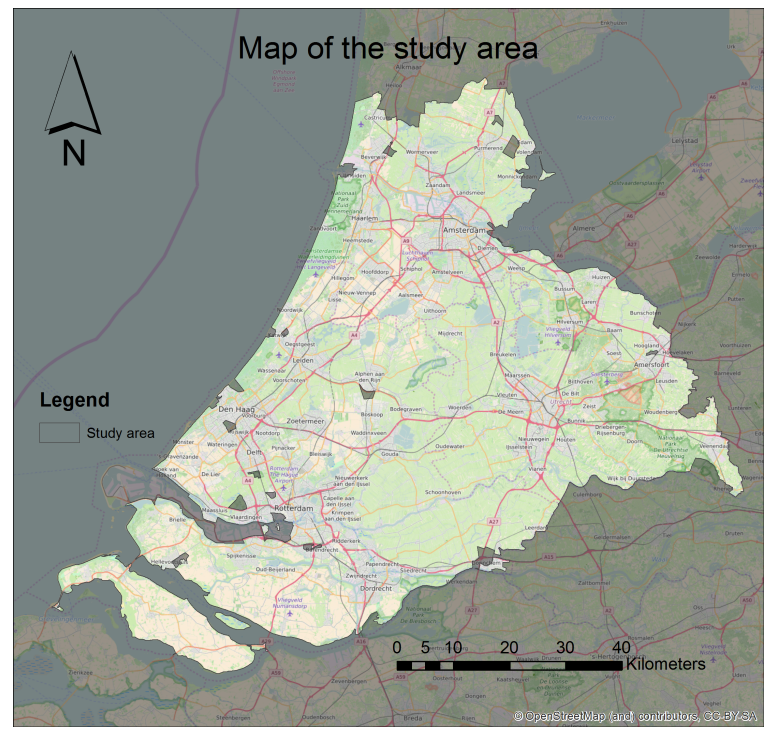


Figure 9: Raw data and sovereign colors.

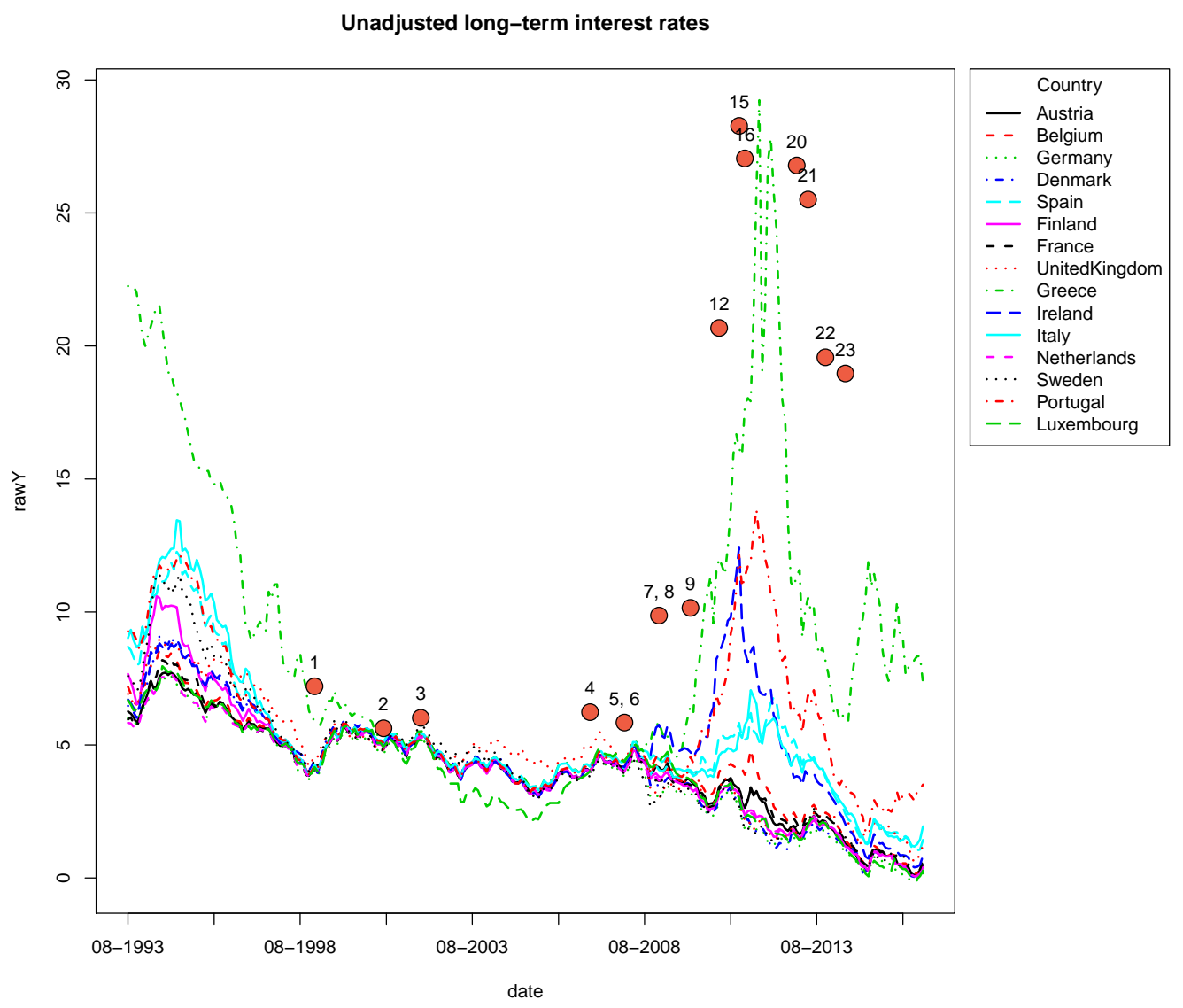


SAR + ARMA (AIC) residuals
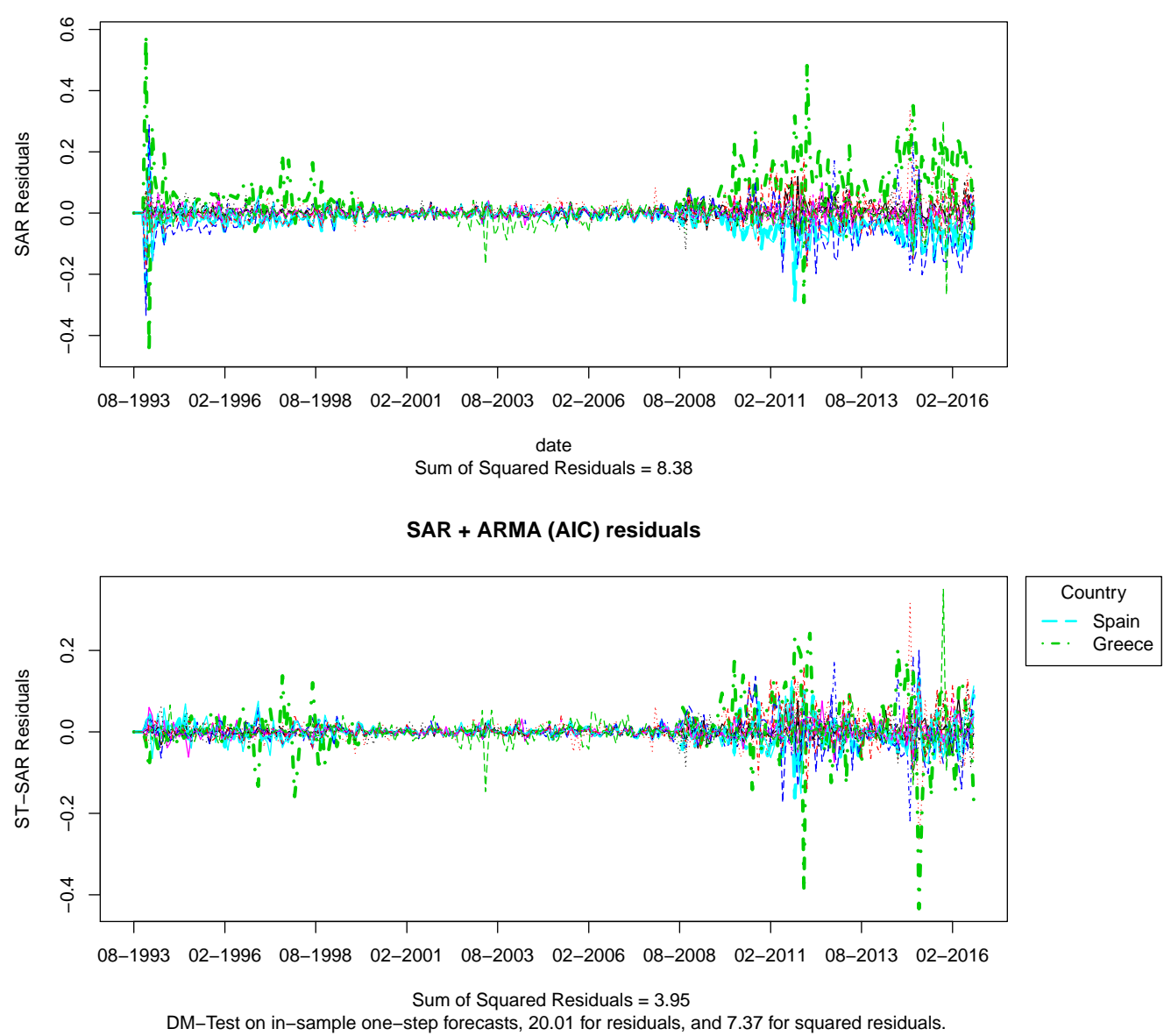

Figure 10: Residuals of the final SAR and ST-SAR models showing that after filtering out linear spatial dynamics, the residuals of Spain and Greece are not properly centered on zero. 

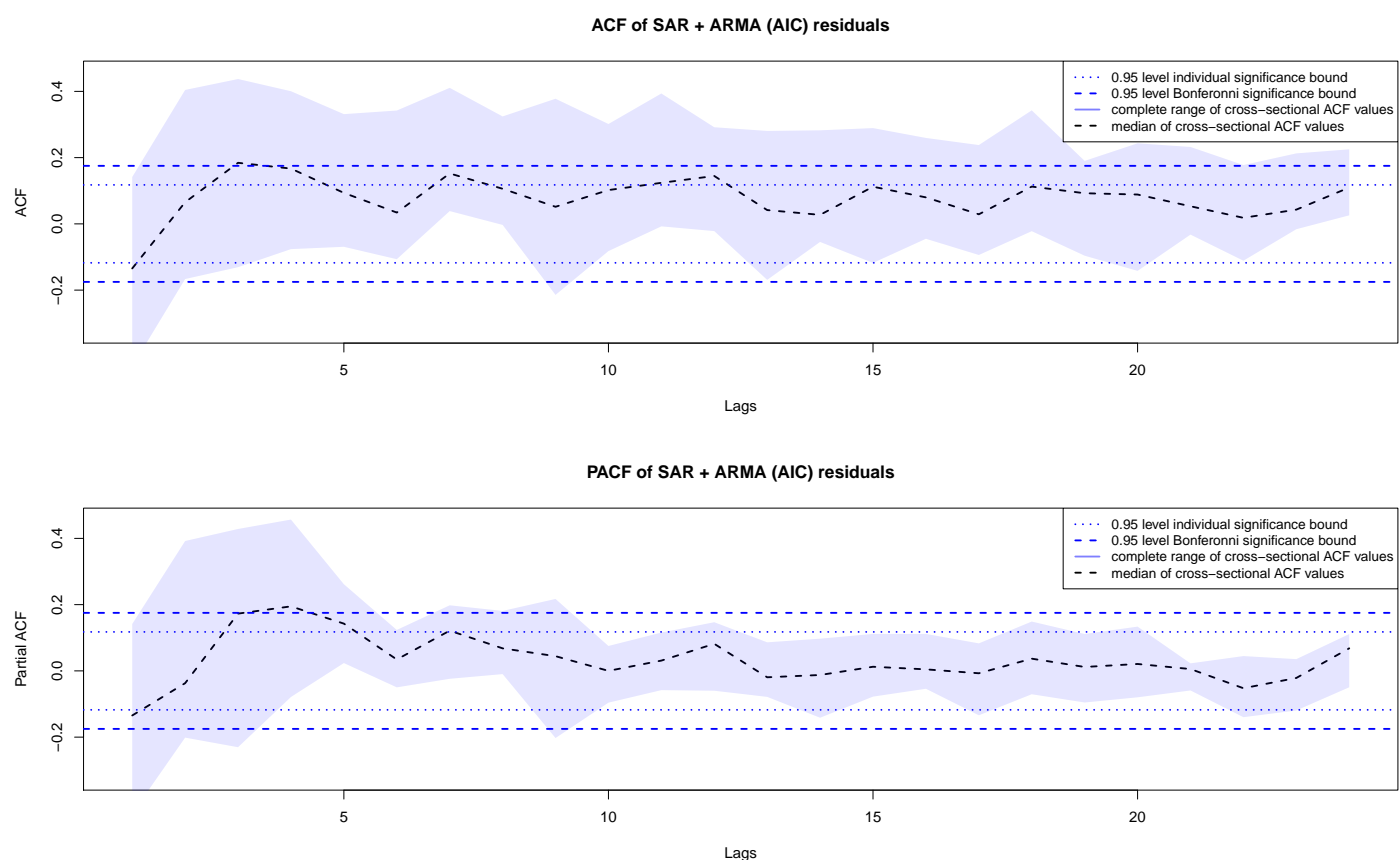

ACF of ST-SAR (AIC) + ARMA (AIC) residuals

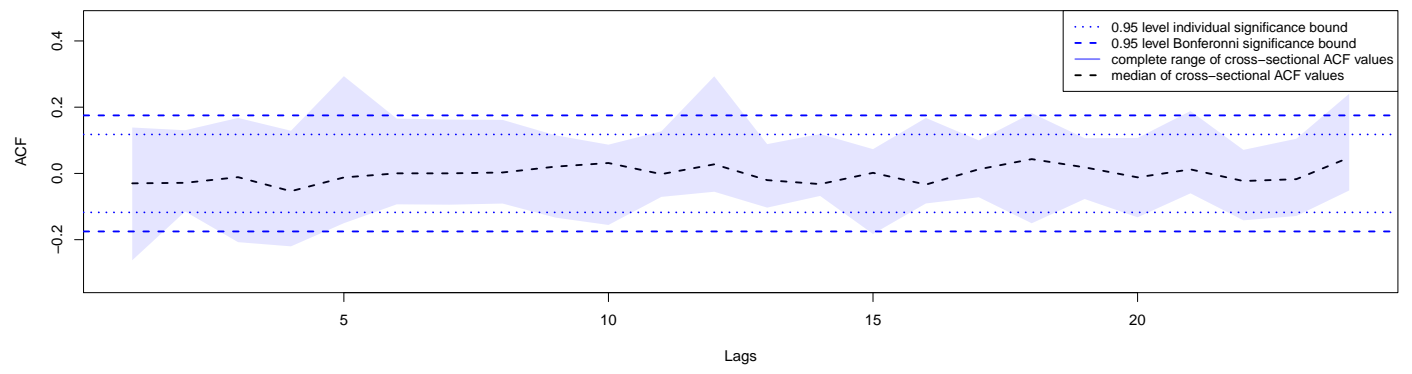

PACF of ST-SAR (AIC) + ARMA (AIC) residuals

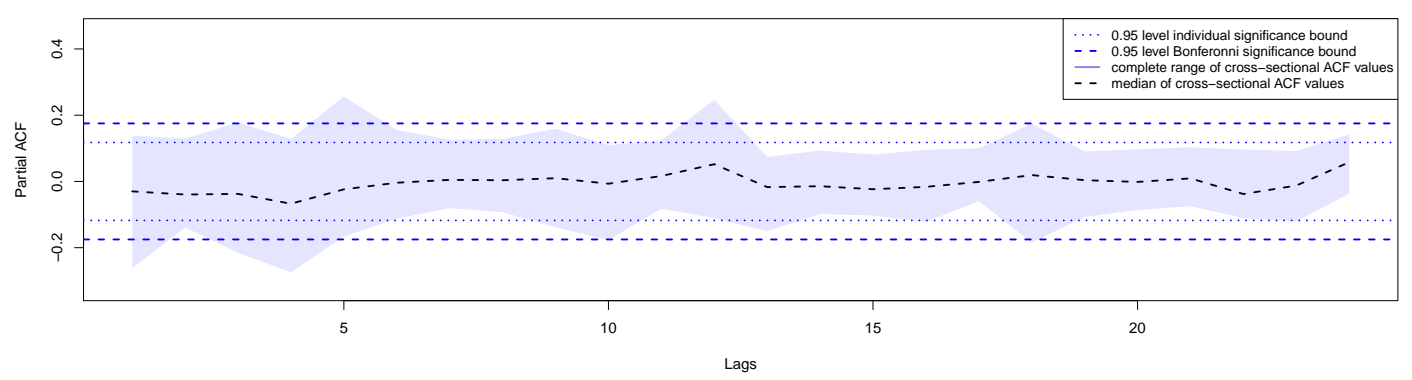

Figure 11: Residual correlations of the SAR and ST-SAR. 


\section{Timeline of events related to European Long term Interest Rates}

1 January 1999, start of the Euro;

2 January 2001, Greece joins the Euro;

3 January 2002, Euro coins and notes are introduced;

4 January 2007, Slovenia joins the Euro;

5 January 2008, Malta and Cyprus join the Euro;

6 November 26, 2008, 200bn European Economic Recovery Plan;

7 January 2009, Slovakia joins the Euro;

8 January 2009, Estonia, Denmark, Latvia and Lithuania join the ERM;

9 December 17, 2009, Greece hits deficit record;

10 April 19, 2010, Greece hits borrowing cost record;

11 May 2, 2010, Greece accepts 110bn bailout package;

12 November 28, 2010, Ireland accepts 85bn bailout package;

13 January 2011, Estonia joins the Euro;

14 February 14, 2011, agreement of 500bn ESM bailout fund;

15 May 3 , 2011, agreement over 78bn bailout package for Portugal;

16 July 21, 2011, agreement over additional 109bn bailout package for Greece;

17 October 6, 2011, Bank of England to inject an additional 75bn pounds into the economy;

18 January 2012, major downgrade wave including nine Eurozone nations by S\&P;

19 June 2012, Spain and Cyprus request assistance from the ESM;

20 January 23 2013, England threats to leave the European Union;

21 May 2, 2013, ECB cuts the rate on its benchmark refinancing facility to $0.50 \%$;

22 November 7, 2013, ECB cuts the rate on its benchmark refinancing facility to $0.25 \%$;

23 June 2014, first negative interest rates by the ECB;

24 June 23, 2016, Brexit. 